\title{
Clinical and virologic characteristics of the first 12 patients with coronavirus disease 2019 (COVID-19) in the United States
}

\author{
The COVID-19 Investigation Team ${ }^{\star}$
}

Data on the detailed clinical progression of COVID-19 in conjunction with epidemiological and virological characteristics are limited. In this case series, we describe the first 12 US patients confirmed to have COVID-19 from 20 January to 5 February 2020, including 4 patients described previously ${ }^{1-3}$. Respiratory, stool, serum and urine specimens were submitted for SARS-CoV-2 real-time reverse-transcription polymerase chain reaction (rRT-PCR) testing, viral culture and whole genome sequencing. Median age was 53 years (range: 21-68); 8 patients were male. Common symptoms at illness onset were cough $(n=8)$ and fever $(n=7)$. Patients had mild to moderately severe illness; seven were hospitalized and demonstrated clinical or laboratory signs of worsening during the second week of illness. No patients required mechanical ventilation and all recovered. All had SARS-CoV-2 RNA detected in respiratory specimens, typically for 2-3 weeks after illness onset. Lowest real-time PCR with reverse transcription cycle threshold values in the upper respiratory tract were often detected in the first week and SARS-CoV-2 was cultured from early respiratory specimens. These data provide insight into the natural history of SARS-CoV-2. Although infectiousness is unclear, highest viral RNA levels were identified in the first week of illness. Clinicians should anticipate that some patients may worsen in the second week of illness.

Twelve patients with confirmed COVID-19 were identified in six states. Five did not require hospitalization and were isolated at home (patients 1-5) and seven were hospitalized for clinical and public health reasons (patients 6-12) (Fig. 1). Median age was 53 years (range: 21-68); eight patients were male (Table 1). Four of five patients with underlying medical conditions were hospitalized (Table 1,2).

Dates of illness onset ranged from 14 to 29 January 2020. Ten patients traveled to mainland China in the 2 weeks before illness onset, including nine to Wuhan City. Two patients were contacts of US COVID-19 patients in this series ${ }^{2}$. Among all patients, the duration of potential exposure ranged from $5 \mathrm{~d}$ to over 1 month; time between last date of possible exposure and illness onset ranged $0-5 \mathrm{~d}$.

The most commonly reported initial signs or symptoms were cough $(n=8)$ and fever $(n=7)$ (Table 1$)$. Two patients reported neither fever nor cough initially, though they developed them subsequently: one reported sore throat as their initial symptom and the other reported diarrhea (one day before fever and cough); the patient with diarrhea had recently traveled outside the United States before illness onset and later tested positive for Giardia and Clostridiodes difficile.

Over the course of illness, patients reported cough $(n=12)$, subjective or measured fever $(n=9)$, diarrhea $(n=3)$ and vomiting $(n=2)$. Three patients who never reported fever were never hospitalized and remained on home isolation. Of these, one patient reported only cough and rhinorrhea; one reported only cough, which began before travel to China and did not change from the initial onset until resolution; and one reported cough, chills, fatigue, headache and nausea.

The clinical course for each hospitalized patient is described in the Supplementary Information and Extended Data (Extended Data Figs. 1-7). All hospitalized patients were managed using standard, contact and airborne precautions, including eye protection. Median duration of fever was $9 \mathrm{~d}$ (range: $2-11$ ). Peak body temperature during hospitalization (range: $99.1-102.9^{\circ} \mathrm{F}$ ) occurred at a median of illness day 9 (range: 4-10) (Fig. 2). All hospitalized patients had oxygen saturation $<94 \%$ on room air at some point during their illness, with oxygen saturation nadir (range: 86-93\%) occurring at a median of illness day 12 (range: 4-23) (Fig. 2). Five patients reported difficulty in breathing and four received supplemental oxygen (Table 2 and Fig. 1). Patient 9 required high-flow nasal cannula oxygen supplementation and intensive care monitoring. No patients required mechanical ventilation.

Two patients received a short course $(\leq 3 \mathrm{~d})$ of corticosteroids. Three, including one who received corticosteroids, received the investigational antiviral remdesivir (Gilead Sciences) under expanded access (compassionate use) for a duration of $4-10 \mathrm{~d}$. Following remdesivir initiation, all had transient gastrointestinal symptoms, including nausea, vomiting, gastroparesis or rectal bleeding. No other post-remdesivir symptoms were observed. Patient 9 reported loose stool and rectal bleeding; as noted above, this patient had recently traveled outside the United States before illness onset and their stool later tested positive for Giardia and Clostridiodes difficile. Remdesivir was discontinued after improvement in each patient's respiratory symptoms.

Blood cultures were negative in all six hospitalized patients tested, including those obtained from four patients treated empirically for bacterial pneumonia. Molecular testing for influenza A and $\mathrm{B}$ on respiratory specimens was negative and multipathogen respiratory PCR panels were negative for all targets in all hospitalized patients (Table 2).

Six of seven hospitalized patients had leukopenia $(<4,000$ cells per $\mu \mathrm{l})$ and the white blood cell count nadir occurred at a median of illness day 9 (range: 4-15) (Fig. 2). Procalcitonin levels were $<0.25 \mathrm{ng} \mathrm{ml}^{-1}$ in all six patients tested. Aminotransferase levels were elevated in all hospitalized patients: aspartate aminotransferase (AST) levels peaked (median peak value $129 \mathrm{Ul}^{-1}$, range 46-190 $\mathrm{Ul}^{-1}$ ) at a median of illness day 13 (range 7-19) and alanine aminotransferase (ALT) levels peaked (median peak value $136 \mathrm{Ul}^{-1}$, 


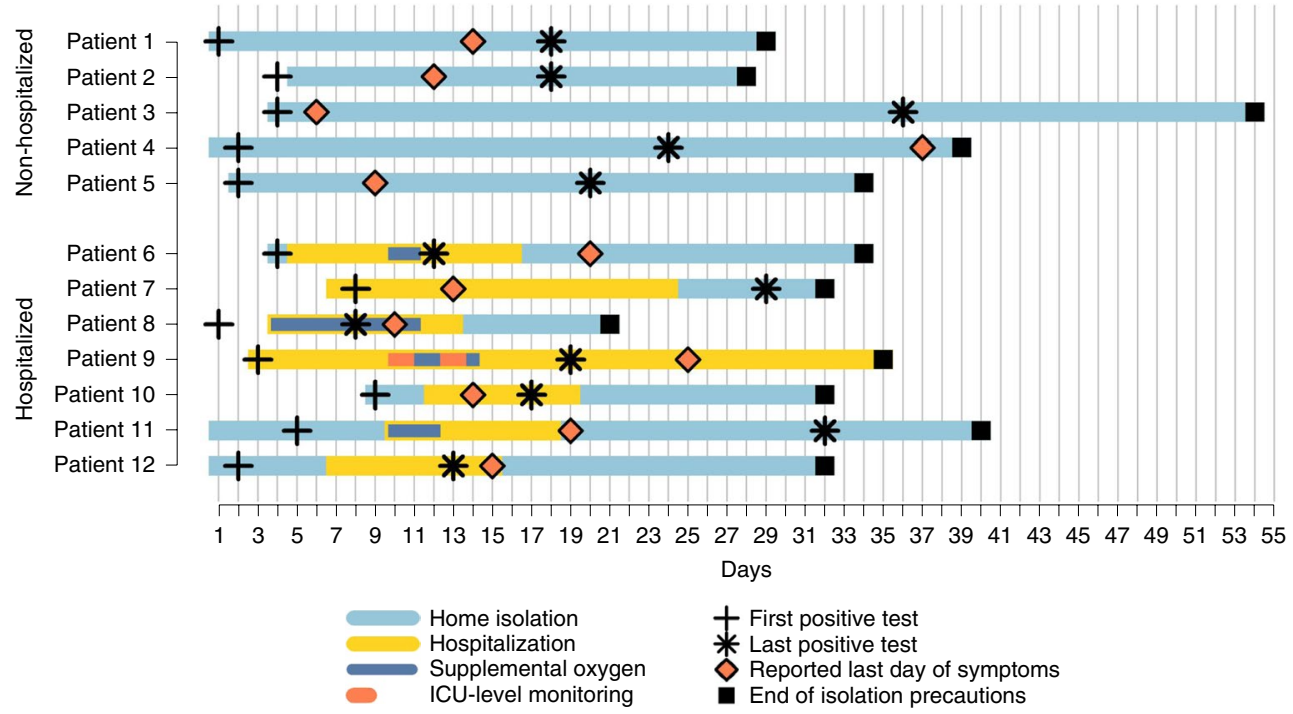

Fig. 1 | Timeline of illness onset, SARS-CoV-2 RNA detection, hospitalization, oxygen requirement and reported symptom resolution among the first 12 patients with COVID-19 in the United States, January to February 2020. Patients 1-5 were not hospitalized and patients 6-12 were hospitalized. Days are sequential from day of symptom onset (day 1). Light blue bars indicate time patients were under home isolation. Yellow bars indicate duration of hospitalization. Dark blue bars indicate duration of supplemental oxygen administration in hospital. The orange dashed bar indicates duration of intensive care unit (ICU)-level monitoring for patient 9. The black ' + ' indicates collection date of the earliest sample that tested positive for SARS-CoV-2 by rRT-PCR. The black asterisk indicates collection date of the latest sample tested by CDC with a positive result for SARS-CoV-2 by rRT-PCR. The orange diamond indicates date of last report of symptoms. The black square indicates the last day of isolation precautions. Patient 9 was isolated in a healthcare facility for the full time period; they were discharged from the first healthcare facility on day 27 and subsequently transferred to a second healthcare facility for public health purposes. Patient 1 reported a cough with initial onset in mid-December before the patient traveled to China. The patient reported no change in the cough from the initial onset until reported resolution on day 14. Because onset date was difficult to determine for this patient, we have used date of detection as day 1 to assess viral RNA detection.

range $\left.66-389 \mathrm{Ul}^{-1}\right)$ at a median of illness day 14 (range: 6-23). Three of seven hospitalized patients had mildly elevated alkaline phosphatase levels $>115 \mathrm{Ul}^{-1}$ (maximum value $163 \mathrm{Ul}^{-1}$ ). Elevated lactate dehydrogenase levels $>600 \mathrm{Ul}^{-1}$, coinciding with clinical deterioration, were observed in two patients tested. No major elevations in serum total bilirubin (seven patients tested) or prolongations in prothrombin time (four patients tested) were identified. Among the three remdesivir recipients, aminotransferase elevation developed in patient $6,1 \mathrm{~d}$ after starting remdesivir and in patient $8,4 \mathrm{~d}$ after starting remdesivir. Patient 9 had an aminotransferase peak before starting remdesivir and a second peak $5 \mathrm{~d}$ after starting remdesivir.

Unilateral or bilateral opacities were seen on chest imaging for all hospitalized patients (Table 2). Four hospitalized patients had normal initial chest radiographs (illness day range: 4-9). One had an abnormal chest computed tomography scan on the day of the normal chest radiograph.

All 12 patients had initial respiratory specimens collected between illness days 1-9 (median, day 4) and all but one tested positive in $\geq 2$ respiratory specimen types (Fig. 3). Viral culture was attempted on initial respiratory specimens from nine patients and was successful for all nine, including two patients who were not hospitalized (Fig. 3); viable SARS-CoV-2 was cultured at day 9 of illness (patient 10), but was not attempted on later specimens. SARS-CoV-2 real-time PCR with reverse transcription (rRT-PCR) cycle threshold $(\mathrm{Ct})$ values of virus isolated from the first tissue culture passage were 12.3-35.7 and for one patient, virus isolated from tissue culture passage 3 had a titer of $7.75 \times 10^{6}$ median tissue culture infectious dose per $\mathrm{ml}$ (Supplementary Table 1); these data were likely more reflective of growth in tissue culture than patient viral load.

Overall, 448 specimens were collected from the 12 patients throughout the course of illness and tested at the US Centers for Disease Control and Prevention (CDC). All 12 patients had
SARS-CoV-2 RNA detected in at least one nasopharyngeal (NP) swab, 11 of 12 in an oropharyngeal (OP) swab, 6 of 6 in sputum, 1 of 11 in serum, 7 of 10 in stool and 0 of 10 in urine (Fig. 3). Among 117 pairs of simultaneous NP and OP specimens, 45 (38\%) had discordant results. Among 32 discordant pairs with one positive specimen, the NP specimen was positive in $21(66 \%)$. Thirteen additional discordant pairs had one negative and one inconclusive specimen. SARS-CoV-2 RNA was detected at a maximum of day 32 in NP specimens, day 36 in OP specimens, day 29 in sputum and day 25 in stool (Fig. 3). Two patients provided sputum specimens after NP and/or OP specimens tested negative and sputum continued to be positive in both patients. In patient 7, viral RNA was detected in sputum $17 \mathrm{~d}$ after the last positive OP specimen and $\geq 2$ weeks after reported symptom resolution. In seven patients who had SARS-CoV-2 RNA detected in stool, most detections occurred when viral RNA was still detectable in the respiratory tract. Among three patients who reported diarrhea, all had viral RNA detected in stool.

Mean Ct values in positive specimens were 17.0-39.0 for NP, 22.3-39.7 for OP and 24.1-39.4 for stool. Ct values of upper respiratory tract specimens were lower in the first week of illness than the second in most patients (Extended Data Fig. 8); in some patients, low $\mathrm{Ct}$ values continued into the second and third week of illness. SARS-CoV-2 rRT-PCR results turned positive in serum of patient 9 in the second week of illness at the time of rapid clinical deterioration.

$\mathrm{Ct}$ values and duration of RNA detection in the upper respiratory tract did not seem to differ by hospitalization status or oxygen requirement.

All patients reported symptom resolution (Fig. 1). Eleven patients reported cough (often intermittent) as the last symptom. Median symptom duration was $14 \mathrm{~d}$ (range: 6-37). SARS-CoV-2 RNA was detected after reported symptom resolution in 7 of 12 patients, including in NP $(n=6)$, OP $(n=3)$, sputum $(n=1)$ and 
Table 1 | Demographic characteristics, exposure history and clinical characteristics of the first 12 patients with COVID-19 in the United States, January to February 2020

\begin{tabular}{|c|c|c|}
\hline \multirow{2}{*}{ Median age (range) - years } & \multicolumn{2}{|c|}{ Values $(N=12)$} \\
\hline & 53 & $(21-68)$ \\
\hline & $n / N$ & $\%$ \\
\hline Male sex - no. & $8 / 12$ & 67 \\
\hline \multicolumn{3}{|l|}{ State - no. } \\
\hline California & $6 / 12$ & 50 \\
\hline Illinois & $2 / 12$ & 17 \\
\hline Arizona & $1 / 12$ & 8 \\
\hline Massachusetts & $1 / 12$ & 8 \\
\hline Washington & $1 / 12$ & 8 \\
\hline Wisconsin & $1 / 12$ & 8 \\
\hline \multicolumn{3}{|l|}{ Initial source of case identification - no. } \\
\hline Outpatient clinic/urgent care & $4 / 12$ & 33 \\
\hline Emergency department & $4 / 12$ & 33 \\
\hline Health department ${ }^{a}$ & $2 / 12$ & 17 \\
\hline Port of entry & $1 / 12$ & 8 \\
\hline Close contact active monitoring & $1 / 12$ & 8 \\
\hline \multicolumn{3}{|l|}{ Exposure history ( $\leq 14 \mathrm{~d}$ before illness onset) } \\
\hline Travel to mainland China - no. & $10 / 12$ & 83 \\
\hline Travel to Wuhan, Hubei Province, China - no. & $9 / 12$ & 75 \\
\hline Exposure to a confirmed US COVID-19 patient - no. & $2 / 12$ & 17 \\
\hline Underlying medical conditions - no. & $5 / 12$ & 42 \\
\hline Cardiac disease ${ }^{\mathrm{b}}$ - no. & $2 / 12$ & 17 \\
\hline Hypertension - no. & $2 / 12$ & 17 \\
\hline Diabetes mellitus - no. & $1 / 12$ & 8 \\
\hline Chronic lung disease - no. & $1 / 12$ & 8 \\
\hline High cholesterol - no. & $2 / 12$ & 17 \\
\hline Fatty liver disease - no. & $1 / 12$ & 8 \\
\hline Hepatitis B - no. & $1 / 12$ & 8 \\
\hline Current tobacco use - no. & $1 / 12$ & 8 \\
\hline \multicolumn{3}{|l|}{ Symptoms reported at illness onset ${ }^{\mathrm{c}}$ - no. } \\
\hline Fever & $7 / 12$ & 58 \\
\hline Subjective & $5 / 7$ & 71 \\
\hline Measured $\left(\geq 100.4^{\circ} \mathrm{F}\right.$ or $\left.38^{\circ} \mathrm{C}\right)$ & $2 / 7$ & 29 \\
\hline Cough $^{d}$ & $8 / 12$ & 67 \\
\hline Fatigue & $5 / 12$ & 42 \\
\hline Shortness of breath (dyspnea) & $1 / 12$ & 8 \\
\hline Sore throat & $1 / 12$ & 8 \\
\hline Headache & $3 / 12$ & 25 \\
\hline Runny nose (rhinorrhea) & $1 / 12$ & 8 \\
\hline Chills & $1 / 12$ & 8 \\
\hline Diarrhea & $1 / 12$ & 8 \\
\hline Nausea & $1 / 12$ & 8 \\
\hline Days of first sample collection, median (range) $(n=12)$ & 4 & $(1-9)$ \\
\hline \multicolumn{3}{|l|}{ Highest level of healthcare utilization - no. } \\
\hline Outpatient clinic or urgent care & $2 / 12$ & 17 \\
\hline Emergency department & $3 / 12$ & 25 \\
\hline Hospitalized & $7 / 12$ & 58 \\
\hline Abnormal chest radiograph - no. & $7 / 9$ & 78 \\
\hline
\end{tabular}

ancludes one person who was a contact of a confirmed COVID-19 patient. Includes coronary artery disease and pacemaker for bradycardia. Reports of hypertension were not included. Initial symptoms were obtained through patient interview and may have differed from symptoms at the time of identification. ${ }^{\mathrm{d}}$ One patient reported a cough with initial onset in mid-December before the patient traveled to China. The patient reported no change in the cough from the initial onset until resolution 2 weeks after SARS-CoV-2 was first detected. stool $(n=3)$ specimens. Home isolation or Transmission-Based Precautions were discontinued for all patients per CDC criteria ${ }^{4}$; the last respiratory specimens tested at CDC with a positive test result were collected from these patients on days 8-36 (median $=$ day 19).

Complete genome sequences were generated from respiratory specimens from all 12 patients. The sequences had $>99 \%$ nucleotide identity to 85 reference sequences of SARS-CoV-2 genomes; phylogenetic tree analysis identified a few distinct subgroups (Extended Data Fig. 9) that were not divergent from each other, suggesting that these patients were identified during an early stage of the outbreak.

We describe the first 12 patients with confirmed COVID-19 in the United States, including clinical course of the first 7 hospitalized patients. Ten patients had traveled to China, including nine to Wuhan City and two had close contact with a US COVID-19 patient. Illness ranged from mild to moderately severe and hospitalized patients showed signs of clinical worsening in the second week. All patients recovered and three patients tolerated treatment with the investigational antiviral remdesivir. SARS-CoV-2 RNA was detected in upper and lower respiratory specimens, stool and serum. The highest viral RNA levels in the upper respiratory tract were typically detected in the first week of illness, and viable SARS-CoV-2 was cultured from early respiratory specimens. Viral RNA was detected after reported symptom resolution for seven patients, although the implications for infectiousness and transmission later in illness are unclear. SARS-CoV-2 genome sequencing and phylogenetic analysis from these 12 patients' respiratory tract specimens support a recent zoonotic transmission event and subsequent human-to-human transmission.

The clinical manifestations described here reflect the milder end of the full disease spectrum. Severe illness and death has since been reported in the United States ${ }^{5-7}$. Among hospitalized patients in this report, the second week of illness was characterized by clinical or laboratory signs of worsening including hypoxemia or elevation of aminotransferases. Although some patients received empiric antibiotics for possible secondary bacterial pneumonia, we found definitive evidence of bacterial co-infection. Worsening in the second week of illness is consistent with previous reports ${ }^{8,9}$ and highlights the importance of close monitoring beyond the first week, even in patients with mild illness or no initial radiographic abnormalities.

Patient 9, the most severely ill among this series, experienced clinical deterioration late in the second week of illness. This was the only patient with SARS-CoV-2 RNA detected in serum and detection in serum was temporally related to clinical deterioration. Similar observations have been described previously ${ }^{10,11}$. Increased proinflammatory cytokines have been observed in patients with COVID $-19^{8}$ and it is possible that cytokine dysregulation and endothelial dysfunction contribute to both clinical worsening and SARS-CoV-2 RNA detection in serum.

We detected viral RNA and cultured virus from upper respiratory specimens, even from patients with predominantly lower respiratory tract illness. Ct values in upper respiratory tract specimens typically were lowest during the first week of illness (suggesting high RNA levels), consistent with previous reports ${ }^{11-14}$. SARS-CoV-2 RNA was detected in upper respiratory tract specimens for 2-3 weeks in most patients and for as long as $36 \mathrm{~d}$. Sputum specimens were less frequently available; in two patients with a productive cough and available sputum, viral RNA was detected in sputum longer than in NP or OP specimens. We detected SARS-CoV-2 RNA in stool of multiple patients and in the serum of one hospitalized patient.

SARS-CoV-2 RNA levels and duration of RNA detection in the upper respiratory tract did not seem to vary by illness severity and viral RNA was detected after reported symptom resolution in several patients. More data are needed to better understand how duration of RNA detection, RNA levels and presence of viable virus are related to symptom progression, illness severity and infectiousness. 


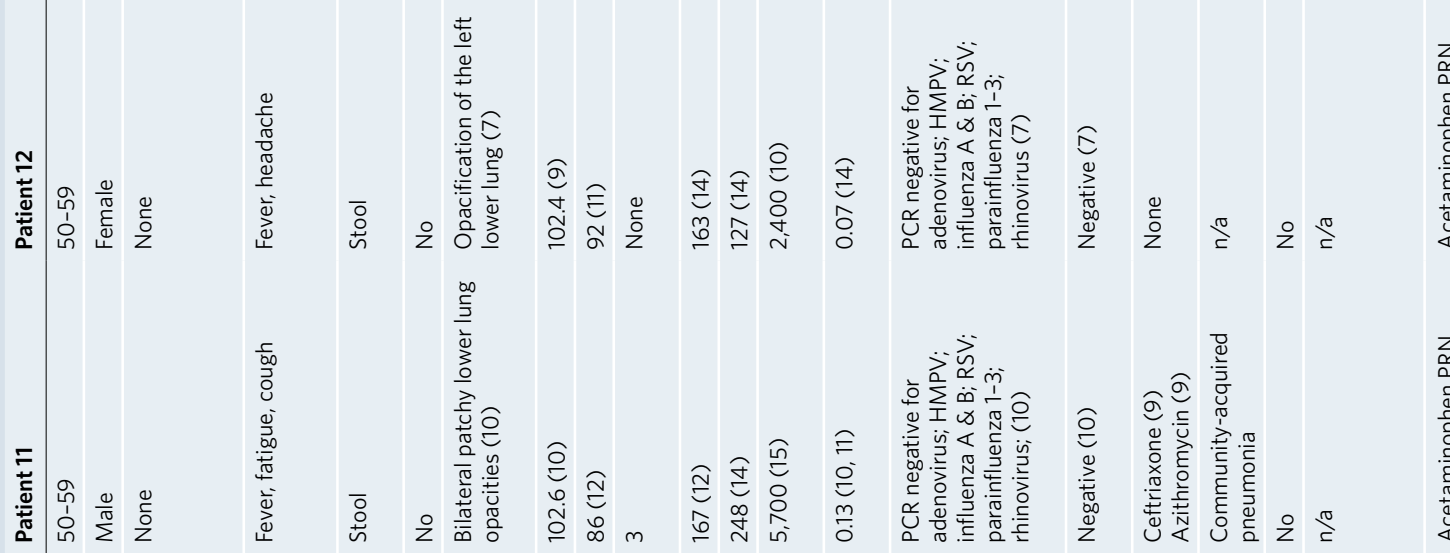
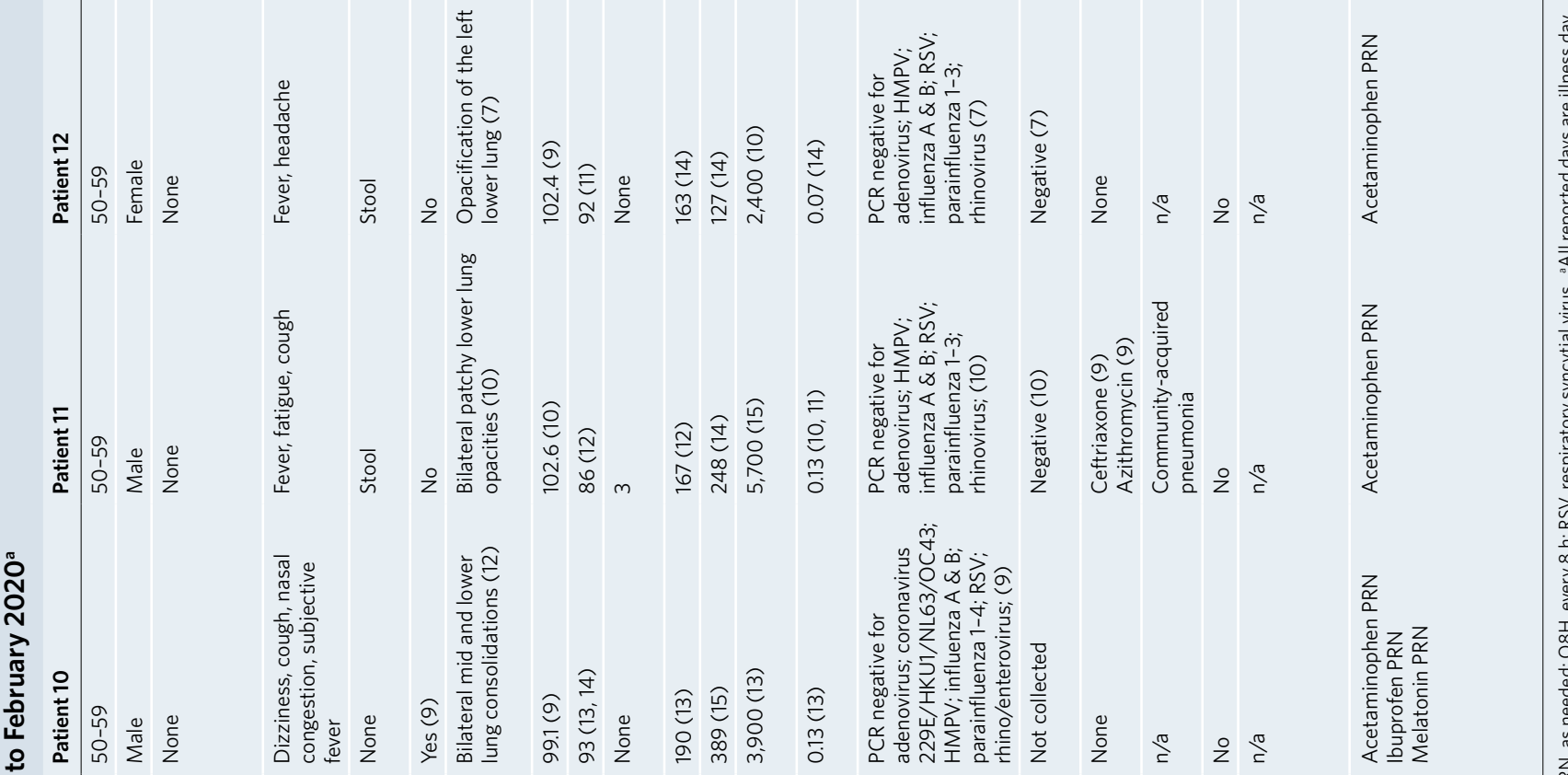

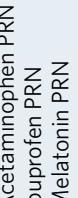
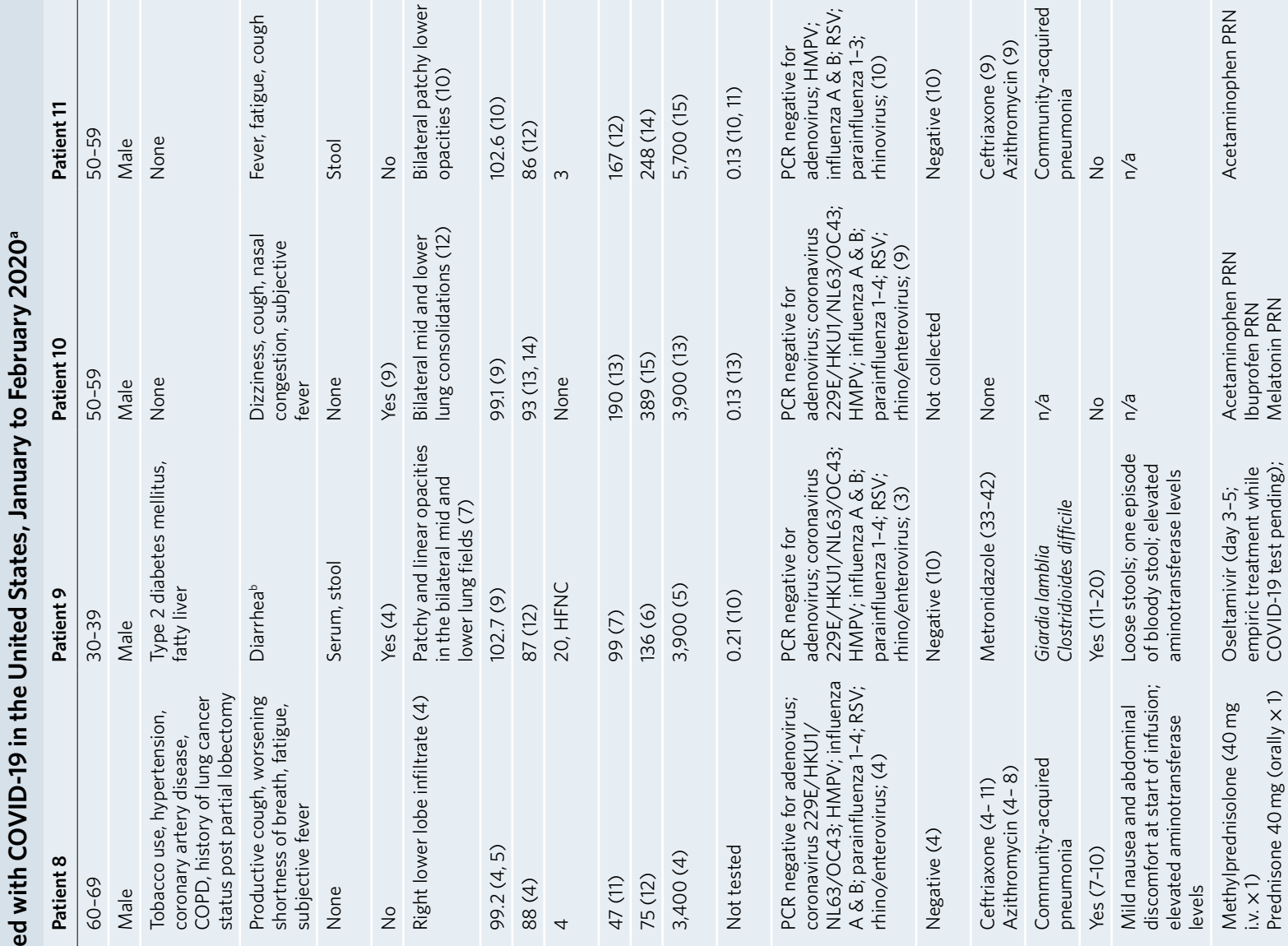

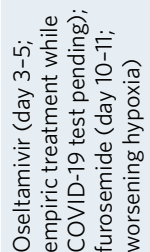

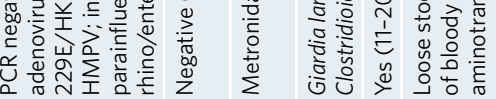
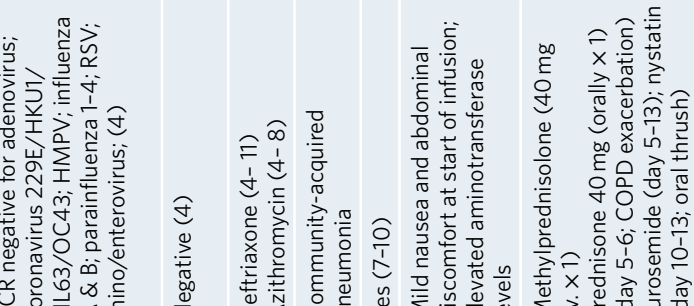

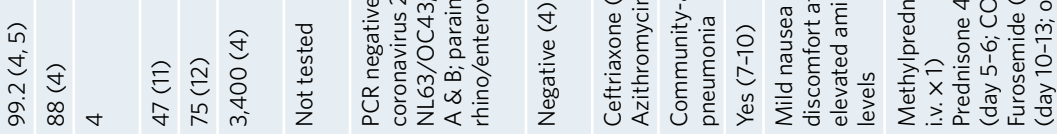<smiles>C1CCC1</smiles>

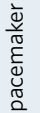

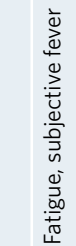

迹京芒

政

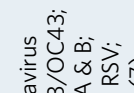

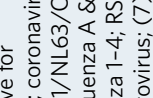

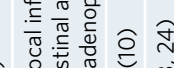

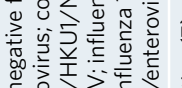

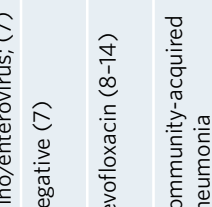

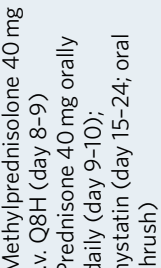
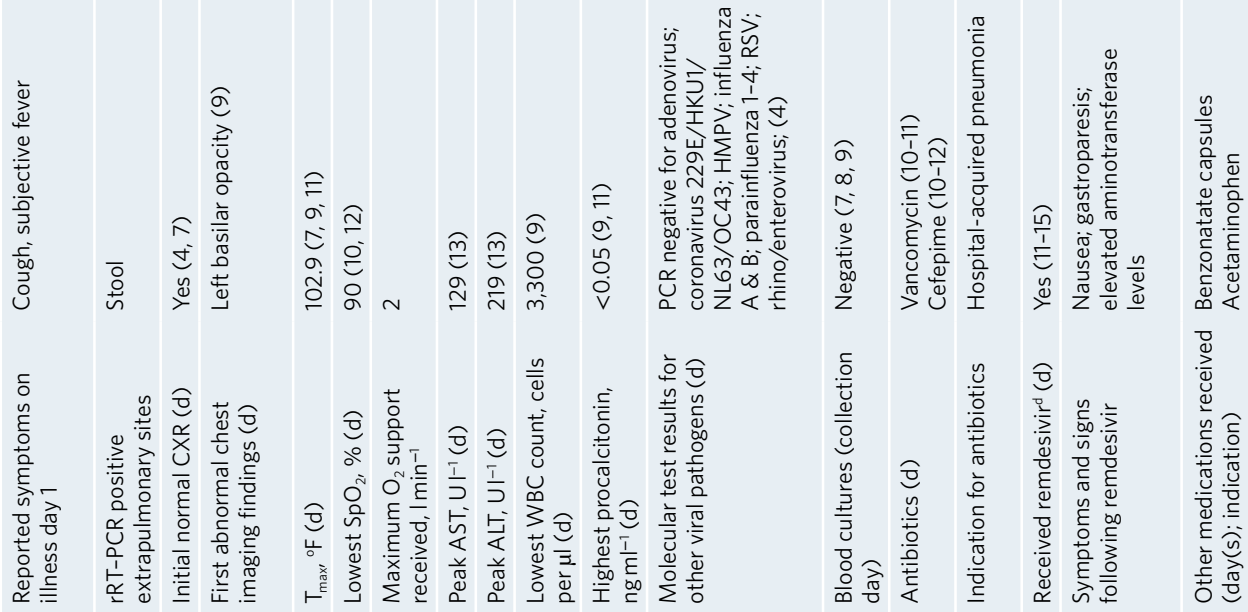

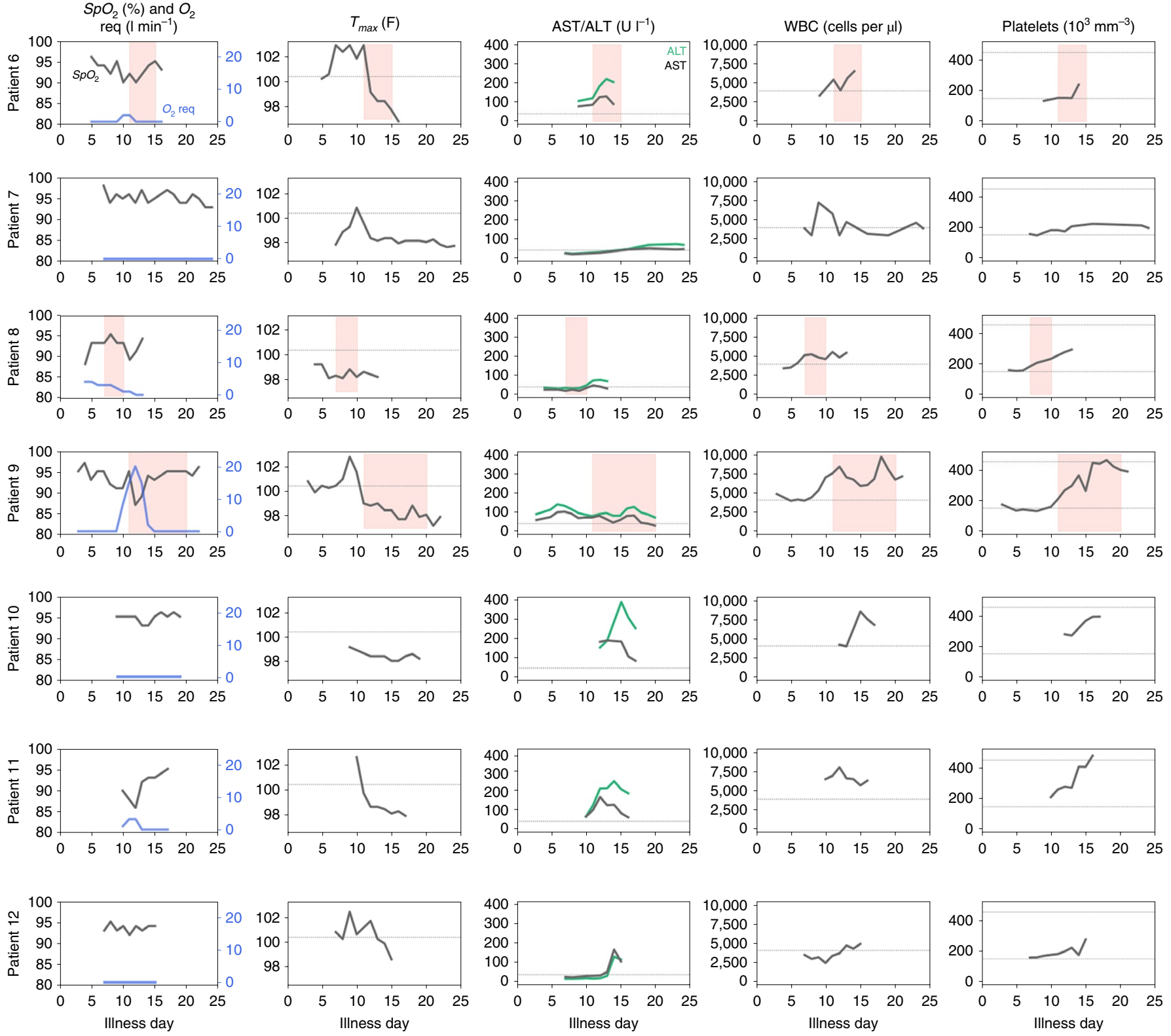

Fig. 2 | Clinical and laboratory values by illness day for the first seven patients hospitalized with COVID-19 in the United States, January to February 2020. Clinical and laboratory values collected during hospitalization are shown for seven hospitalized patients by illness day. Values include oxygen saturation $\left(\mathrm{SpO}_{2}\right)$, supplemental oxygen requirement $\left(\mathrm{O}_{2}\right.$ req), maximum body temperature $\left(\mathrm{T}_{\max }\right)$, AST, ALT and white blood cell (WBC) count. Pink shading indicates days of remdesivir administration for three patients. Dotted lines show fever threshold of $100.4^{\circ} \mathrm{F}\left(\mathrm{T}_{\max }\right), 40 \mathrm{UI}^{-1}$ for $\mathrm{AST}$ and ALT, 4,000 cells per $\mu \mathrm{l}$ for WBC and 150 and $25010^{3} \mathrm{~mm}^{-3}$ for platelets.

Three hospitalized patients received the investigational antiviral remdesivir under expanded access (compassionate use) at the time of clinical worsening based upon a decision by each patient's clinician. Remdesivir inhibits viral replication through premature termination of RNA transcription ${ }^{15,16}$. In vitro studies have demonstrated that remdesivir inhibits SARS-CoV-2 replication in nonhuman cells ${ }^{17}$. Because remdesivir use was not given as part of a randomized controlled trial, we are unable to assess effectiveness or safety. Randomized controlled trials of remdesivir are underway ${ }^{18-20}$. Two hospitalized patients received corticosteroids. The World Health Organization and CDC advise against use of corticosteroids unless indicated for another reason ${ }^{21}$.

Our investigation has several limitations. Our patient sample is small, and results may not be generalizable. Information from patient interviews may have been subject to response bias. The threshold for hospitalization in these early cases was likely low because of uncertainty about COVID-19 clinical course. Illness resolution dates may be imprecise due to nonspecific lingering symptoms or symptoms from chronic or unrelated conditions. Clinical laboratory tests and radiographic studies were ordered as a part of routine patient care and were not collected systematically. SARS-CoV-2 RNA detection does not necessarily reflect the presence of infectious virus and rRT-PCR Ct values may have varied due to specimen collection or handling.

Characterization of the first 12 COVID-19 patients identified in the United States provides insight into the epidemiology, clinical characteristics and natural history of SARS-CoV-2 infection. Although duration of infectiousness is unclear, these early data 

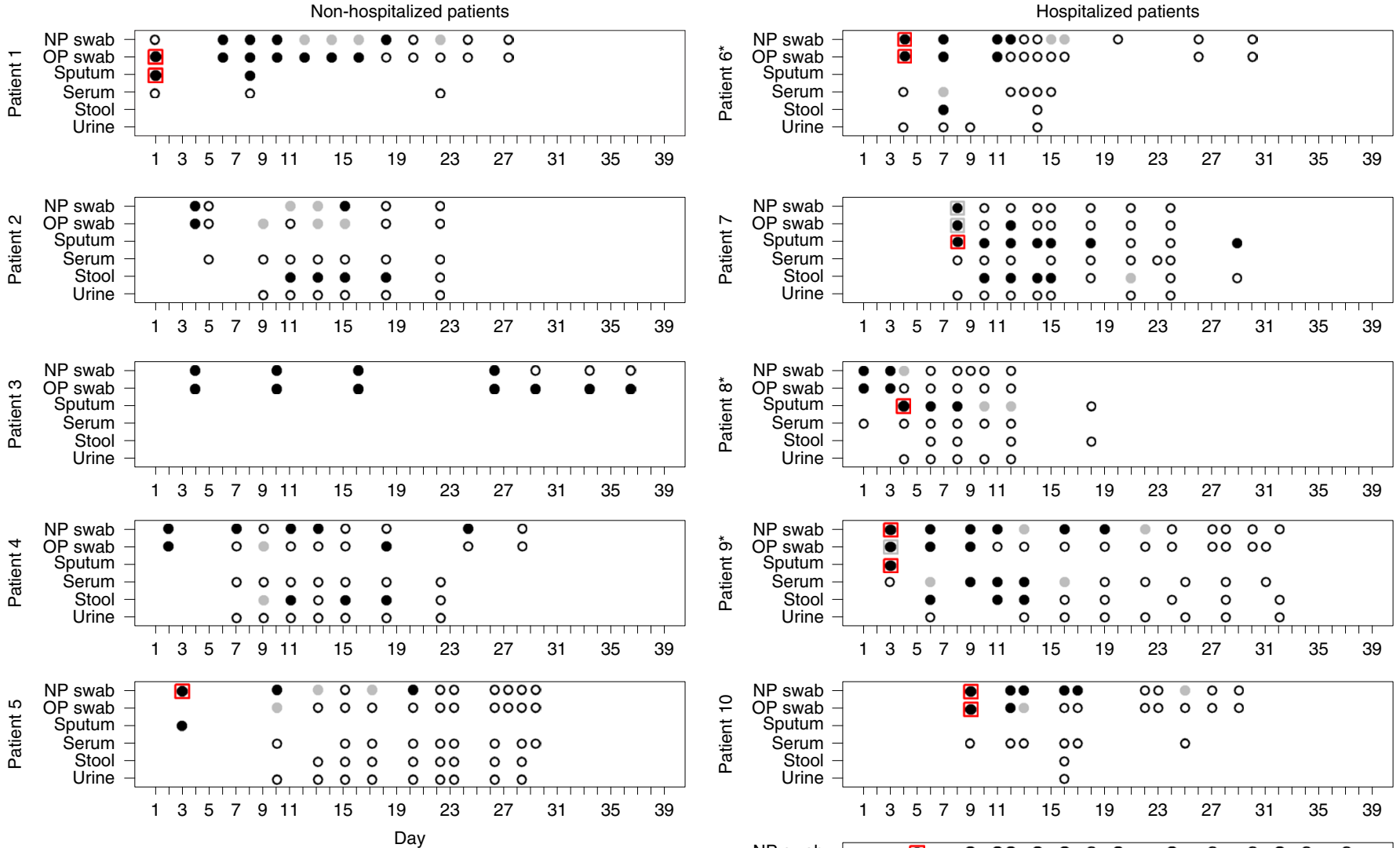

- rtRT-PCR positive a Virus isolation successful

o rtRT-PCR negative Virus isolation not successful

rtRT-PCR inconclusive * Received supplemental oxygen

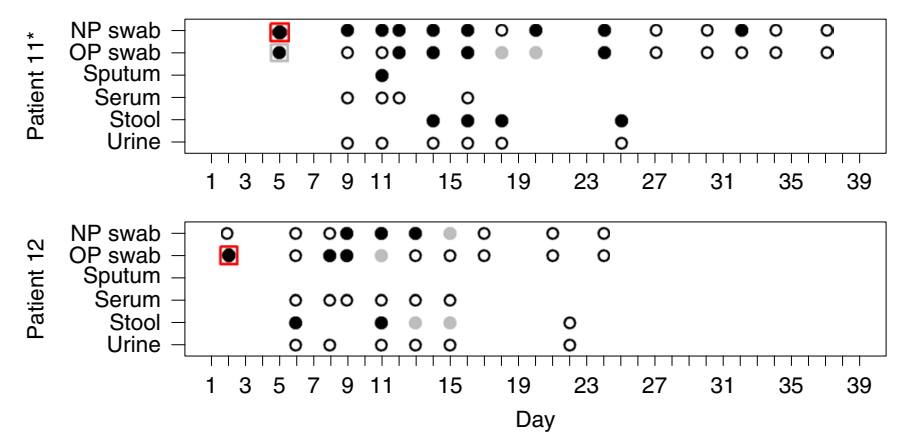

Fig. 3 | SARS-CoV-2 rRT-PCR results by specimen type and day among the first 12 patients with COVID-19 in the United States, January to February 2020. Specimen types tested include NP swab, OP swab, sputum, serum, stool and urine. Days are sequential from day of symptom onset (day 1). Viral culture was attempted on selected respiratory specimens collected early in the course of illness. rRT-PCR results were reported as positive (all three targets positive), negative (all three targets negative) or inconclusive (only one or two positive targets). Black-filled circles indicate rRT-PCR-positive specimens. Black-outlined circles indicate rRT-PCR-negative specimens. Gray-filled circles indicate specimens with inconclusive rRT-PCR results. Red squares surrounding black-filled circles indicate rRT-PCR-positive specimens from which viral culture was successful. Gray squares surrounding black-filled circles indicate rRT-PCR-positive specimens from which viral culture was unsuccessful. An asterisk indicates patients who required supplemental oxygen.

show viable virus can be cultured readily from upper respiratory tract specimens soon after illness onset; further studies on infectious period and risk factors for transmission are needed. Clinicians should anticipate that some patients may worsen in the second week of illness, but appropriate monitoring of these patients will present challenges as healthcare systems work to meet the increasing demands. Studies are urgently needed to better characterize risk factors for and early indicators of severe disease. Randomized controlled trials of therapeutic options and their effects on clinical outcomes and infectiousness are critical to guide clinical and public health management. Additional investigations to understand clinical course, immunological response, SARS-CoV-2 RNA detection, viral culture and transmission, will inform clinical management and public health strategies to prevent the spread of disease.

\section{Online content}

Any methods, additional references, Nature Research reporting summaries, source data, extended data, supplementary information, acknowledgements, peer review information; details of author contributions and competing interests; and statements of data and code availability are available at https://doi.org/10.1038/s41591020-0877-5.

Received: 14 March 2020; Accepted: 6 April 2020;

Published online: 23 April 2020

\section{References}

1. Holshue, M. L. et al. First case of 2019 novel coronavirus in the United States. N. Engl. J. Med. 382, 929-936 (2020). 
2. Ghinai, I. et al. First known person-to-person transmission of severe acute respiratory syndrome coronavirus 2 (SARS-CoV-2) in the USA. Lancet 395, 1137-1144 (2020).

3. Scott, S. E. et al. First mildly ill, non-hospitalized case of coronavirus disease 2019 (COVID-19) without viral transmission in the United States-Maricopa County, Arizona, 2020. Clin. Infect. Dis. https://doi. org/10.1093/cid/ciaa374 (2020).

4. Centers for Disease Control and Prevention. Disposition of non-hospitalized patients with COVID-19. https://www.cdc.gov/coronavirus/2019-ncov/hcp/ disposition-in-home-patients.html (2020).

5. Arentz, M. et al. Characteristics and outcomes of 21 critically ill patients with COVID-19 in Washington state. JAMA https://doi.org/10.1001/ jama.2020.4326 (2020).

6. McMichael, T. M. et al. COVID-19 in a long-term care facility-King County, Washington, February 27-March 9, 2020. Preprint at Morb. Mortal. Wkly. Rep. https://www.cdc.gov/mmwr/volumes/69/wr/mm6912e1.htm (2020).

7. CDC COVID-19 Response Team. Severe outcomes among patients with coronavirus disease 2019 (COVID-19) - United States, February 12-March 16, 2020. Preprint at Morb. Mortal. Wkly. Rep. https://doi.org/10.15585/ mmwr.mm6912e2 (2020).

8. Huang, C. et al. Clinical features of patients infected with 2019 novel coronavirus in Wuhan, China. Lancet 395, 497-506 (2020).

9. Wang, D. et al. Clinical characteristics of 138 hospitalized patients with 2019 novel coronavirus-infected pneumonia in Wuhan, China. JAMA https://doi. org/10.1001/jama.2020.1585 (2020).

10. Chen, W. et al. Detectable 2019-nCoV viral RNA in blood is a strong indicator for the further clinical severity. Emerg. Microbes Infect. 9, 469-473 (2020).

11. Kim, J. Y et al. Viral load kinetics of SARS-CoV-2 infection in first two patients in Korea. J. Korean Med. Sci. 35, e86 (2020).
12. Pan, Y., Zhang, D., Yang, P., Poon, L. L. M. \& Wang, Q. Viral load of SARS-CoV-2 in clinical samples. Lancet Infect. Dis. 20, 411-412 (2020).

13. Zou, L. et al. SARS-CoV-2 viral load in upper respiratory specimens of infected patients. N. Engl. J. Med. 382, 1177-1179 (2020).

14. Wölfel, R. et al. Virological assessment of hospitalized patients with COVID-2019. Nature https://doi.org/10.1038/s41586-020-2196-x (2020).

15. Mulangu, S. et al. A randomized, controlled trial of Ebola virus disease therapeutics. N. Engl. J. Med. 381, 2293-2303 (2019).

16. Warren, T. K. et al. Therapeutic efficacy of the small molecule GS-5734 against Ebola virus in rhesus monkeys. Nature 531, 381-385 (2016).

17. Wang, M. et al. Remdesivir and chloroquine effectively inhibit the recently emerged novel coronavirus (2019-nCoV) in vitro. Cell Res. 30, 269-271 (2020).

18. ClinicalTrials.gov. Severe 2019-nCoV Remdesivir RCT. February 6, 2020 https://clinicaltrials.gov/ct2/show/NCT04257656 (2020).

19. ClinicalTrials.gov. Adaptive COVID-19 Treatment Trial. March 12, 2020 https:/clinicaltrials.gov/ct2/show/NCT04280705?term=adaptive\&con $\mathrm{d}=$ COVID-19\&draw $=2 \& \operatorname{rank}=1(2020)$.

20. ClinicalTrials.gov. Mild/Moderate 2019-nCoV Remdesivir RCT. February 5, 2020 https://clinicaltrials.gov/ct2/show/NCT04252664?cond=remdesivir\&dra $\mathrm{w}=2$ \&rank=2 (2020).

21. World Health Organization. Clinical management of severe acute respiratory infection when novel coronavirus $(2019-\mathrm{nCoV})$ infection is suspected: interim guidance. 28 January 2020 (WHO, 2020).

Publisher's note Springer Nature remains neutral with regard to jurisdictional claims in published maps and institutional affiliations.

This is a U.S. government work and not under copyright protection in the U.S.; foreign copyright protection may apply 2020

The COVID-19 Investigation Team

Stephanie A. Kujawski', Karen K. Wong', Jennifer P. Collins', Lauren Epstein', Marie E. Killerby', Claire M. Midgley' ${ }^{1 凶}$, Glen R. Abedi', N. Seema Ahmed ${ }^{2}$, Olivia Almendares', Francisco N. Alvarez ${ }^{3}$, Kayla N. Anderson', Sharon Balter ${ }^{4}$, Vaughn Barry', Karri Bartlett ${ }^{5}$, Karlyn Beer', Michael A. Ben-Aderet ${ }^{6}$, Isaac Benowitz', Holly M. Biggs', Alison M. Binder', Stephanie R. Black ${ }^{7}$, Brandon Bonin ${ }^{8}$, Catherine H. Bozio', Catherine M. Brown ${ }^{9}$, Hollianne Bruce ${ }^{10}$, Jonathan Bryant-Genevier', Alicia Budd', Diane Buell"1, Rachel Bystritsky³, Jordan Cates', E. Matt Charles ${ }^{12}$, Kevin Chatham-Stephens', Nora Chea', Howard Chiou ${ }^{4}$, Demian Christiansen ${ }^{13}$, Victoria Chu', Sara Cody ${ }^{8}$, Max Cohen', Erin E. Conners', Aaron T. Curns', Vishal Dasari', Patrick Dawson', Traci DeSalvo ${ }^{14}$, George Diaz ${ }^{15}$, Matthew Donahue', Suzanne Donovan ${ }^{16}$, Lindsey M. Duca', Keith Erickson ${ }^{15}$, Mathew D. Esona', Suzanne Evans"11, Jeremy Falk6, Leora R. Feldstein', Martin Fenstersheib ${ }^{17}$, Marc Fischer', Rebecca Fisher ${ }^{4}$, Chelsea Foo ${ }^{4}$, Marielle J. Fricchione ${ }^{7}$, Oren Friedman ${ }^{6}$, Alicia Fry', Romeo R. Galang ${ }^{1}$, Melissa M. Garcia"1, Susan I. Gerber', Graham Gerrard"1", Isaac Ghinai ${ }^{1,2}$, Prabhu Gounder4, Jonathan Grein ${ }^{6}$, Cheri Grigg1, Jeffrey D. Gunzenhauser ${ }^{4}$, Gary I. Gutkin ${ }^{6}$, Meredith Haddix ${ }^{4}$, Aron J. Hall', George S. Han ${ }^{8}$, Jennifer Harcourt ${ }^{1}$, Kathleen Harriman ${ }^{18}$, Thomas Haupt ${ }^{14}$, Amber K. Haynes ${ }^{1}$, Michelle Holshue ${ }^{1,19}$, Cora Hoover ${ }^{18}$, Jennifer C. Hunter ${ }^{1}$, Max W. Jacobs ${ }^{1}$, Claire Jarashow ${ }^{4}$, Kiran Joshi ${ }^{13}$, Talar Kamali ${ }^{4}$, Shifaq Kamili', Lindsay Kim ${ }^{1}$, Moon Kim ${ }^{4}$, Jan King ${ }^{4}$, Hannah L. Kirking', Amanda Kita-Yarbro5, Rachel Klos ${ }^{14}$, Miwako Kobayashi', Anna Kochariann ${ }^{14}$, Kenneth K. Komatsu ${ }^{20}$, Ram Koppaka', Jennifer E. Layden ${ }^{7}$, Yan Li', Scott Lindquist ${ }^{19}$, Stephen Lindstrom ${ }^{1}$, Ruth Link-Gelles', Joana Lively', Michelle Livingston ${ }^{11}$, Kelly Lo ${ }^{6}$, Jennifer Lo ${ }^{9}$, Xiaoyan Lu', Brian Lynch', Larry Madoff', Lakshmi Malapati', Gregory Marks ${ }^{6}$, Mariel Marlow', Glenn E. Mathisen ${ }^{16}$, Nancy McClung', Olivia McGovern', Tristan D. McPherson ${ }^{17}$, Mitali Mehta ${ }^{6}$, Audrey Meier ${ }^{15}$, Lynn Mello ${ }^{17}$, Sung-sil Moon', Margie Morgan ${ }^{6}$, Ruth N. Moro', Janna' Murray', Rekha Murthy', Shannon Novosad', 
Sara E. Oliver', Jennifer O'Shea ${ }^{2}$, Massimo Pacilli', Clinton R. Paden', Mark A. Pallansch', Manisha Patel', Sajan Patel ${ }^{3}$, Isabel Pedraza ${ }^{6}$, Satish K. Pillai', Talia Pindyck', Ian Pray 1,14, Krista Queen', Nichole Quick ${ }^{21}$, Heather Reese', Roshan Reporter ${ }^{4}$, Brian Rha', Heather Rhodes', Susan Robinson ${ }^{20}$, Philip Robinson"1 , Melissa A. Rolfes', Janell A. Routh', Rachel Rubin ${ }^{13}$, Sarah L. Rudman ${ }^{8}$, Senthilkumar K. Sakthivel', Sarah Scott',22, Christopher Shepherd ${ }^{6}$, Varun Shetty', Ethan A. Smith ${ }^{6}$, Shanon Smith ${ }^{8}$, Bryan Stierman', William Stoecker ${ }^{2}$, Rebecca Sunenshine ${ }^{22}$, Regina Sy-Santos"1, Azaibi Tamin', Ying Tao', Dawn Terashita ${ }^{4}$, Natalie J. Thornburg', Suxiang Tong', Elizabeth Traub ${ }^{4}$, Ahmet Tural' ${ }^{15}$, Anna Uehara', Timothy M. Uyeki', Grace Vahey', Jennifer R. Verani', Elsa Villarino ${ }^{8}$, Megan Wallace', Lijuan Wang', John T. Watson', Matthew Westercamp', Brett Whitaker', Sarah Wilkerson ${ }^{15}$, Rebecca C. Woodruff', Jonathan M. Wortham', Tiffany Wu', Amy Xie', Anna Yousaf', Matthew Zahn' ${ }^{21}$ and Jing Zhang'

${ }^{1}$ Centers for Disease Control and Prevention, Atlanta, GA, USA. ${ }^{2}$ Metro Infectious Disease Consultants, Hoffman Estates, IL, USA. ${ }^{3}$ University of California

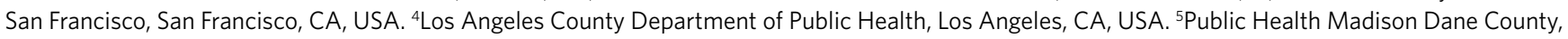
Madison, WI, USA. ${ }^{6}$ Cedars-Sinai Health System, Los Angeles, CA, USA. ${ }^{7}$ Chicago Department of Public Health, Chicago, IL, USA. ${ }^{8}$ Santa Clara County Public Health Department, San Jose, CA, USA. 'Massachusetts Department of Public Health, Boston, MA, USA. ${ }^{10}$ Snohomish Health District, Everett, WA, USA. " ${ }^{11}$ oag Memorial Hospital Presbyterian, Newport Beach, CA, USA. ${ }^{12}$ Illinois Department of Public Health, Springfield, IL, USA. ${ }^{13}$ Cook County Department of Public Health, Oak Forest, IL, USA. ${ }^{14}$ Wisconsin Department of Health Services, Madison, WI, USA. ${ }^{15}$ Providence Regional Medical Center Everett, Everett, WA, USA. ${ }^{16}$ Olive View-UCLA Medical Center, Sylmar, CA, USA. ${ }^{17}$ San Benito County Public Health Services, Hollister, CA, USA. ${ }^{18}$ California Department of Public Health, Richmond, CA, USA. ${ }^{19}$ Washington State Department of Health, Tumwater, WA, USA. ${ }^{20}$ Arizona Department of Health Services, Phoenix, AZ, USA. ${ }^{21}$ Orange County Health Care Agency, Santa Ana, CA, USA. ${ }^{22}$ Maricopa County Department of Public Health,

Phoenix, AZ, USA. 凶e-mail: ydk5@cdc.gov 


\section{Methods}

CDC's Human Research Protection Office determined that this work was exempt from human participants' research regulations as it involved identification, control or prevention of disease in response to an immediate public health threat. Patient consent was waived. Forms were approved under Office of Management and Budget, number 0920-1011.

Local health departments, in consultation with clinicians, identified patients under investigation (PUIs) for COVID-19 beginning 17 January 2020. PUI testing criteria changed during this period but included the presence of fever and/or lower respiratory symptoms (for example, cough or shortness of breath) and at least one epidemiological risk factor in the 2 weeks before symptom onset. Between 17 and 31 January 2020, epidemiological risk factors were travel from Wuhan City, close contact with a patient with laboratory-confirmed COVID-19 or currently under investigation for COVID-19 (refs. ${ }^{22,23}$ ). Beginning 1 February 2020, epidemiological risk factors changed to close contact with a patient with confirmed COVID-19 or history of travel from mainland China ${ }^{24}$. During both time periods, close contact was defined as being within 6 feet for a prolonged period of time ${ }^{25}$ or contact with respiratory secretions ${ }^{26}$. Close contacts of patients with confirmed COVID-19 were identified by local and state health departments and monitored for illness. Specimens from PUIs, including symptomatic close contacts, were tested for SARS-CoV-2 at $\mathrm{CDC}^{27}$

Upper respiratory tract (NP and OP) and available lower respiratory tract (sputum) specimens were collected and tested for SARS-CoV-2 RNA by rRT$\mathrm{PCR}^{28}$. A case of COVID-19 was defined as identification of laboratory-confirmed SARS-CoV- 2 in $\geq 1$ specimen from a patient. We included COVID-19 patients confirmed by CDC between 20 January 2020 and 5 February 2020; the size of this convenience sample was not predetermined and we aimed to describe the first cases as soon as possible. Patients sought outpatient or inpatient care at local facilities; some were transferred or referred to facilities that had specifically prepared to receive patients with COVID-19.

Patients were interviewed by public health officials about demographics, exposures, travel history and symptoms, including signs or symptoms before presentation. For all patients, available medical records were reviewed. For hospitalized patients, clinicians systematically abstracted data from the medical record.

Illness day 1 was defined as the first day of reported COVID-19 signs and symptoms; collection date of the first SARS-CoV-2-positive specimen was used for one patient with no clear symptom onset date. When prehospital symptoms or onset dates in the medical record differed from those reported from the public health interview, the latter were used. Results for virological tests were reported relative to illness day 1. Duration of potential exposure to SARS-CoV-2 was defined as dates of travel to China or dates of first to last exposure to a US patient with COVID-19. Fever was defined as feeling feverish or measuring body temperature $\geq 100.4^{\circ} \mathrm{F}$. We requested collection of NP swabs, OP swabs, sputum (if available), serum, urine and stool every $2-3 \mathrm{~d}$ throughout infection.

Multiple clinical and laboratory observations were included from each of the 12 patients. No associations between clinical or laboratory data and outcomes were tested statistically. Data were analyzed and visualized using Excel in Microsoft Office 365, SAS v.9.4, R v.3.6.2 and Python v.3.7.3 (refs. ${ }^{29-32}$ ).

CDC evaluated specimens using SARS-CoV-2 RNA detection, viral culture, whole genome sequencing and phylogenetic analysis.

All patient specimens were tested for the presence of SARS-CoV-2 RNA by rRT-PCR targeting three regions of the gene encoding the nucleocapsid protein $^{28}$. The rRT-PCR results were reported as positive (all targets positive), negative (all targets negative) or inconclusive (only one or two positive targets). If an initial result was inconclusive, the specimen was re-tested; if both tests were inconclusive, the final result was reported as inconclusive. For inconclusive results, a positive detection of SARS-CoV-2 could not be definitively ruled out. If available, Ct values, which are approximately inversely related to the RNA levels in each specimen ${ }^{33}$, were reported as the mean of three reported Ct values. Results from serial rRT-PCR testing were not immediately available to inform clinical management.

Viral culture was attempted from early SARS-CoV-2-positive respiratory specimens (NP, OP or sputum) with Ct values $<33$. Specifically, $100 \mu \mathrm{l}$ of clinical specimens were diluted twofold across a 96-well plate in serum-free DMEM supplemented with $2 \times$ penicillin-streptomycin and $2 \times$ amphotericin B (Sigma). Vero CCL-81 cells were trypsinized and resuspended in DMEM $+10 \%$ FBS $+2 \times$ penicillin-streptomycin $+2 \times$ amphotericin B at $2.5 \times 105$ cells per ml. A $100-\mu l$ cell suspension was added directly to the clinical specimen dilutions and mixed gently by pipetting. The inoculated cultures were grown in a humidified $37^{\circ} \mathrm{C}$ incubator with $5 \% \mathrm{CO}_{2}$ and observed for cytopathic effect daily. When cytopathic effect was observed, presence of SARS-CoV-2 was confirmed by rRT-PCR.

Nucleic acid was extracted from respiratory specimens (NP, OP or sputum) positive for SARS-CoV-2 rRT-PCR and used for whole genome sequencing on both Sanger and Oxford Nanopore MinION sequencing platforms. For Sanger sequencing, 37 sets of individual nested PCR assays spanning the entire 2019-nCoV genome were designed based on the reference sequence, GenBank accession number NC045512. PCR amplicons were sequenced in both directions using Big Dye 3.1 cycle sequencing kits (Thermo Fisher Scientific) on an ABI 3730 Automated Capillary Sequencer (Thermo Fisher Scientific) with PCR primers and additional internal primers. The consensus sequences were generated from both sequencing directions using Sequencher 5.4.6 (Gene Codes Corporation). For Nanopore sequencing, individual PCR amplicons were pooled and barcoded by sample, which were used for library preparation using the Ligation Sequencing kit (Oxford Nanopore Technologies). Libraries were run on a MinION sequencer and consensus sequences were generated using minimap v.2.17 and samtools v.1.9 (refs. ${ }^{34,35}$ ).

Full genome sequences from the 12 confirmed cases in this report and 85 full genome sequences (as of 11 February 2020) available from GenBank and the Global Initiative on Sharing All Influenza Data database were aligned using MAFFT v.7.450 (ref. ${ }^{36}$ ). Sequences with obvious early stop codons were excluded. Phylogenetic trees were then inferred with the maximum likelihood method using the Hasegawa Kishino Yano nucleotide substitution model with $\gamma$-distributed rate variation among sites $(\mathrm{HKY}+\mathrm{G})$ and 1,000 bootstrap replicates implemented in Geneious Prime (Biomatters) and MEGA X ${ }^{37,38}$.

Reporting Summary. Further information on research design is available in the Nature Research Reporting Summary linked to this article.

\section{Data availability}

Viral sequences generated during the current study are available in the GENBANK repository (GenBank accession numbers: MN997409 (Pt 1), MT044258 (Pt 2), MT039888 (Pt 3), MT027064 (Pt 4), MT039887 (Pt 5), MT020880 (Pt 6), MN988713 (Pt 7), MT044257 (Pt 8), MN994467 (Pt 9), MN994468 (Pt 10), MT027062 (Pt 11), MT027063 (Pt 12); GISAID numbers: 406223 (Pt 1), 410044 (Pt 2), 409067 (Pt 3), 408010 (Pt 4), 408670 (Pt 5), 407214 (Pt 6), 404253 (Pt 7), 410045 (Pt 8), 406034 (Pt 9), 406036 (Pt 10), 408008 (Pt 11) and 408009 (Pt 12)). GISAID sequences can be accessed at www.gisaid.org. Other datasets of clinical and laboratory data presented in the current study may be available from the corresponding author on request.

\section{References}

22. CDC Health Alert Network. Update and Interim Guidance on Outbreak of 2019 Novel Coronavirus (2019-nCoV) in Wuhan, China. January 17, 2020 https://emergency.cdc.gov/han/han00426.asp (2020).

23. Bajema, K. L. et al. Persons evaluated for 2019 novel coronavirus-United States, January 2020. Morb. Mortal. Wkly. Rep. 69, 166-170 (2020).

24. CDC Health Alert Network. Update and Interim Guidance on Outbreak of 2019 Novel Coronavirus (2019-nCoV). February 1, 2020 https://emergency. cdc.gov/han/han00427.asp (2020).

25. Centers for Disease Control and Prevention. Interim US Guidance for Risk Assessment and Public Health Management of Healthcare Personnel with Potential Exposure in a Healthcare Setting to Patients with Coronavirus Disease 2019 (COVID-19). https://www.cdc.gov/coronavirus/2019-ncov/hcp/ guidance-risk-assesment-hcp.html (2020).

26. Centers for Disease Control and Prevention. Evaluating and Reporting Persons Under Investigation (PUI). https://www.cdc.gov/ coronavirus/2019-nCoV/hcp/clinical-criteria.html (2020).

27. Centers for Disease Control and Prevention. Interim Guidelines for Collecting, Handling, and Testing Clinical Specimens from Persons Under Investigation (PUIs) for Coronavirus Disease 2019 (COVID-19). https://www. cdc.gov/coronavirus/2019-nCoV/lab/guidelines-clinical-specimens.html.

28. Holshue, M. L. et al. First case of 2019 novel coronavirus in the United States. N. Engl. J. Med. 382, 929-936 (2020).

29. Hunter, J. D. Matplotlib: a 2D graphics environment. Comput. Sci. Eng. 9 , 90-95 (2007).

30. van Rossum, G. Python tutorial. Technical Report CS-R9526 (Centrum voor Wiskunde en Informatica (CWI), 1995).

31. R Core Team. R: A Language and Environment for Statistical Computing ( $\mathrm{R}$ Foundation for Statistical Computing, 2019).

32. Waskom, M. et al. mwaskom/seaborn: v.0.10.0. https://doi.org/10.5281/ zenodo.883859.

33. Patrinos, G. P. \& Ansorge, W. Molecular Diagnostics (Elsevier, 2010). https:// doi.org/10.1016/C2009-0-01736-X

34. Li, H. Minimap2: pairwise alignment for nucleotide sequences. Bioinformatics 34, 3094-3100 (2018)

35. Li, H. et al. The sequence alignment/map format and SAMtools. Bioinformatics 25, 2078-2079 (2009).

36. Katoh, K. \& Standley, D. M. MAFFT multiple sequence alignment software version 7: Improvements in performance and usability. Mol. Biol. Evol. 30, 772-780 (2013).

37. Kumar, S., Stecher, G., Li, M., Knyaz, C. \& Tamura, K. MEGA X: molecular evolutionary genetics analysis across computing platforms. Mol. Biol. Evol. 35, 1547-1549 (2018)

38. Posada, D. jModelTest: phylogenetic model averaging. Mol. Biol. Evol. 25, 1253-1256 (2008) 


\section{Acknowledgements}

We acknowledge the following individuals for their valuable contribution to field investigations of cases: L. Hicks, P. Lauro and A. Peterson Pompa from Arizona Department of Health Services; A. Dratch and E. Shearer from Orange County Health Care Agency; and A. Aguiar, A. Alvarez, V. Guerra, C. Marttila, P. Mottu-Monteon and M. White from San Benito County Public Health Services. We also acknowledge the following individuals from the US CDC for their valuable contribution to this work: S. Aggarwal, A. Cleveland, R. Dahl, W. Edens, J. Leung, E. Shearer, M. Prill, N. Clemmons, C. Hoff, C. Mattison, F. Whitehill, J. Farrar, A. Kambhampati, A. Newton, S. Smith-Jeffcoat and N. Weise for data or specimen collection and management; J. Anderson for data visualization; H.-Y. Chang, H. Chien, M. Cohen, R. Gautam, L. Jenkins, N. Kamal, M. Rogan, J. Thomas, S. York, E. Breaker, D. Campbell, J. Daniels, C. Elkins, M. Karlsson, M. Keckler, A. Laufer Halpin, D. Lonsway, A. Lyons, M. Martinez-Smith, G. McAllister, S. McAllister, J. Noble-Wang, A. Perry, J. Rasheed, N. Reese, A. Shams, L. Spicer and J. Whitworth for diagnostic testing; and M. Freedman and B. Manns for operational support of the CDC COVID-19 Clinical Team. The findings and conclusions in this report are those of the authors and do not necessarily represent the official position of the CDC.

\section{Author contributions}

All authors meet authorship criteria and approve of publication. C.M.M. and K.K.W. had full access to all data in the study and take responsibility for the integrity of data and accuracy of data analysis. C.M.M., S.A.K., A.J.H., A.F., S.I.G., J.T.W., S.T., N.J.T. and S.L. contributed to the concept and design of the investigation and follow-up of all patients to describe epidemiological, clinical and virological characteristics; K.K.W., J.P.C., L.E.,
R.R.G and T.M.U. contributed to the concept and design of the in-depth clinical investigation of hospitalized patients. All authors contributed to the acquisition, analysis or interpretation of data. S.A.K., K.K.W, J.P.C, L.E., M.E.K. and C.M.M. drafted the manuscript. All authors contributed to critical revision of the manuscript for important intellectual content. S.A.K., K.K.W., M.E.K., G.R.A, A.U. and C.M.M. contributed to statistical analysis and data visualization. C.M.M., A.F., A.J.H., M.A.R., S.T., N.J.T. and S.L. contributed to the supervision of the investigation and follow-up of all patients to describe epidemiological, clinical and virological characteristics; T.M.U. supervised the in-depth clinical investigation of hospitalized patients.

\section{Competing interests}

The authors declare no competing interests.

\section{Additional information}

Extended data is available for this paper at https://doi.org/10.1038/s41591-020-0877-5. Supplementary information is available for this paper at https://doi.org/10.1038/ s41591-020-0877-5.

Correspondence and requests for materials should be addressed to C.M.M.

Peer review information Alison Farrell and João Monteiro are the primary editors on this article and managed its editorial process and peer review in collaboration with the rest of the editorial team.

Reprints and permissions information is available at www.nature.com/reprints. 


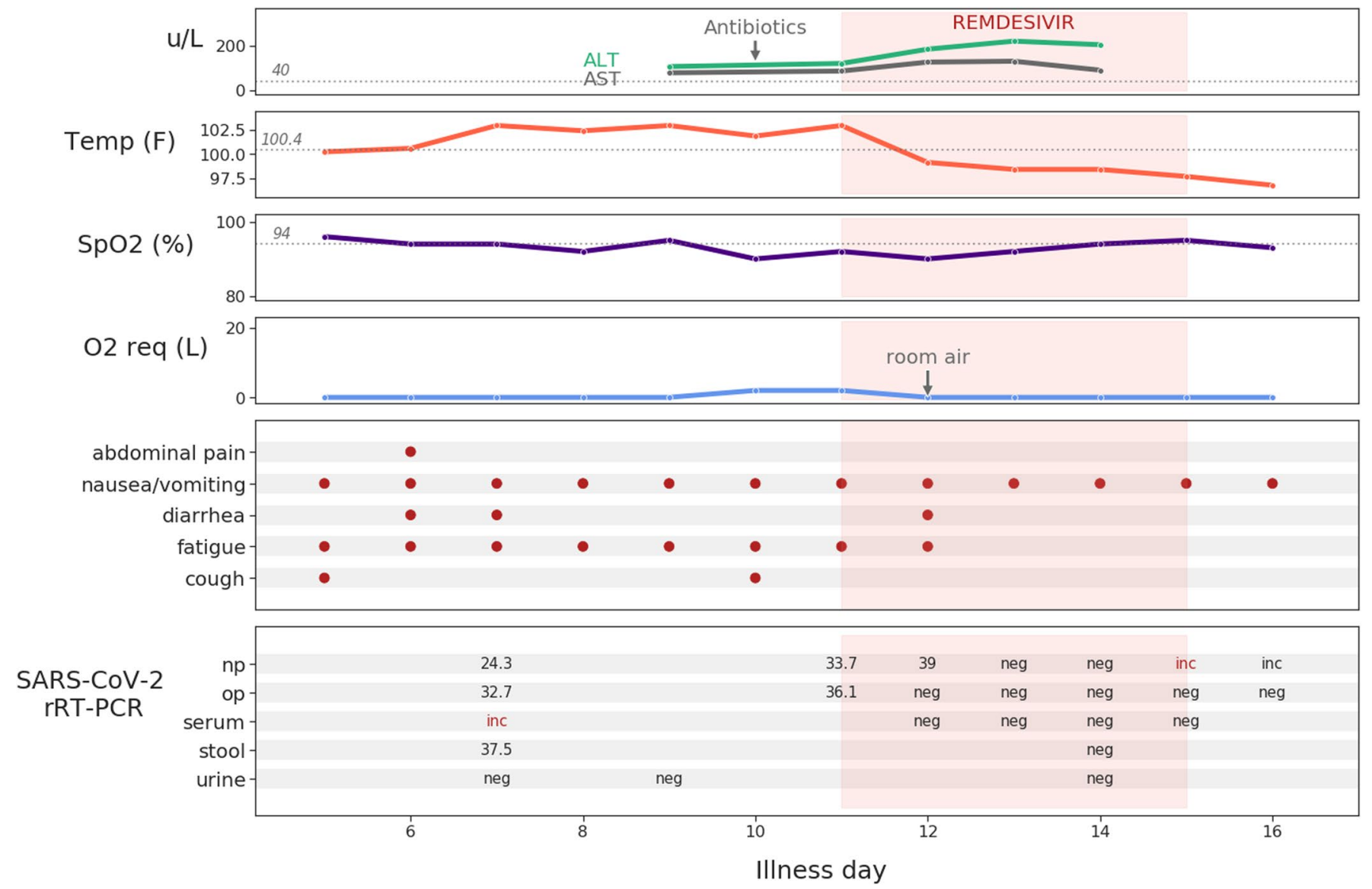

Extended Data Fig. 1 | Patient 6 laboratory and clinical values, symptoms, and SARS-CoV-2 rRT-PCR results during hospitalization. Aminotransferase levels, maximum daily body temperature, minimum daily oxygen saturation, maximum daily oxygen requirement, symptoms, and rRT-PCR results are shown for hospitalization. Pink shading indicates remdesivir administration. Cycle threshold (Ct) values are shown for positive rRT-PCR tests. Abbreviations: AST, aspartate aminotransferase; ALT, alanine aminotransferase; SpO2, oxygen saturation; op, oropharyngeal; np, nasopharyngeal; neg, negative; inc, inconclusive. 


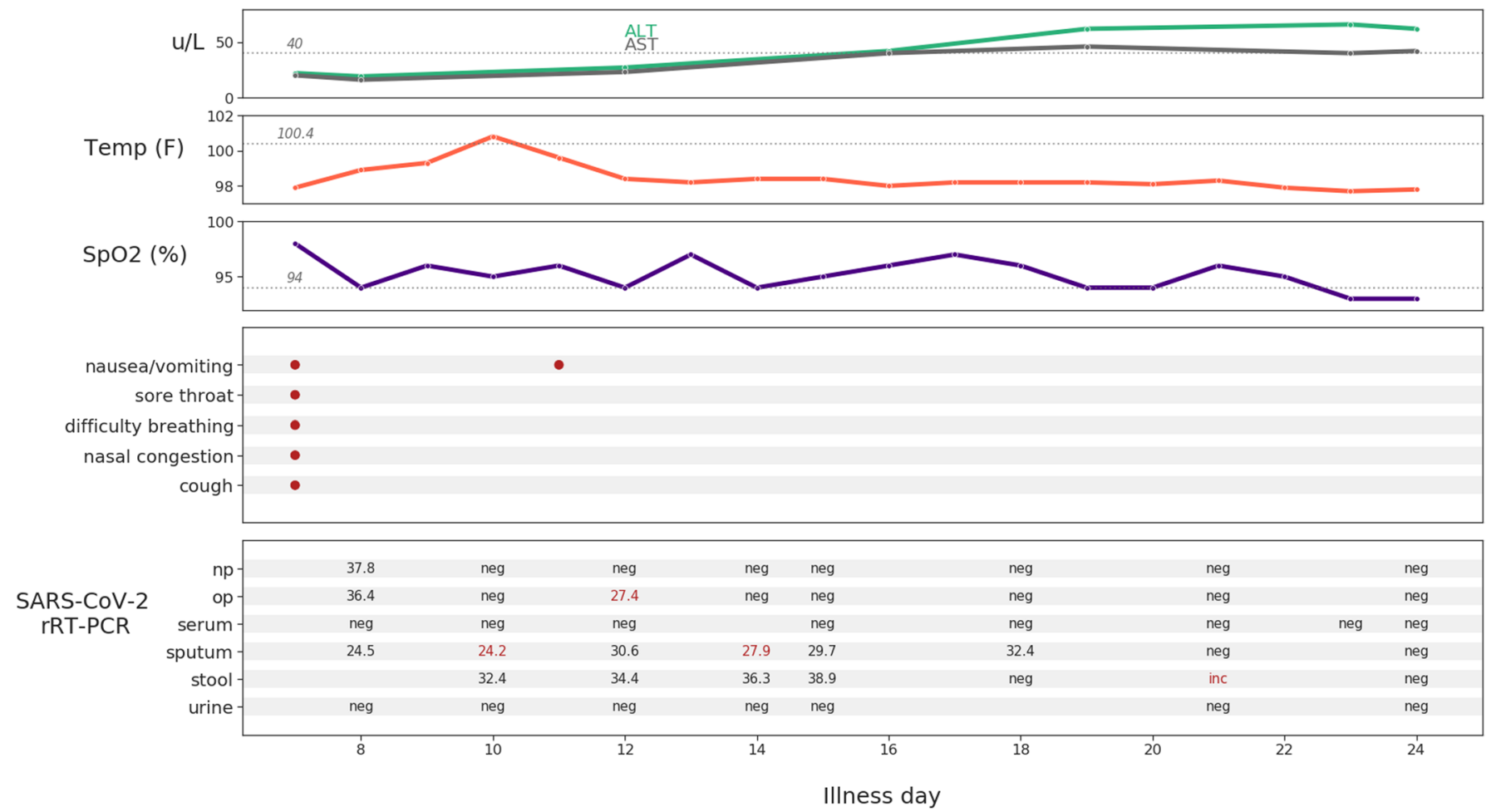

Extended Data Fig. 2 | Patient 7 laboratory and clinical values, symptoms, and SARS-CoV-2 rRT-PCR results during hospitalization. Aminotransferase levels, maximum daily body temperature, minimum daily oxygen saturation, symptoms, and rRT-PCR results are shown for hospitalization. Cycle threshold $(\mathrm{Ct})$ values are shown for positive rRT-PCR tests. Red text indicates decreasing Ct values. Abbreviations: AST, aspartate aminotransferase; ALT, alanine aminotransferase; SpO2, oxygen saturation; op, oropharyngeal; np, nasopharyngeal; neg, negative; inc, inconclusive. 


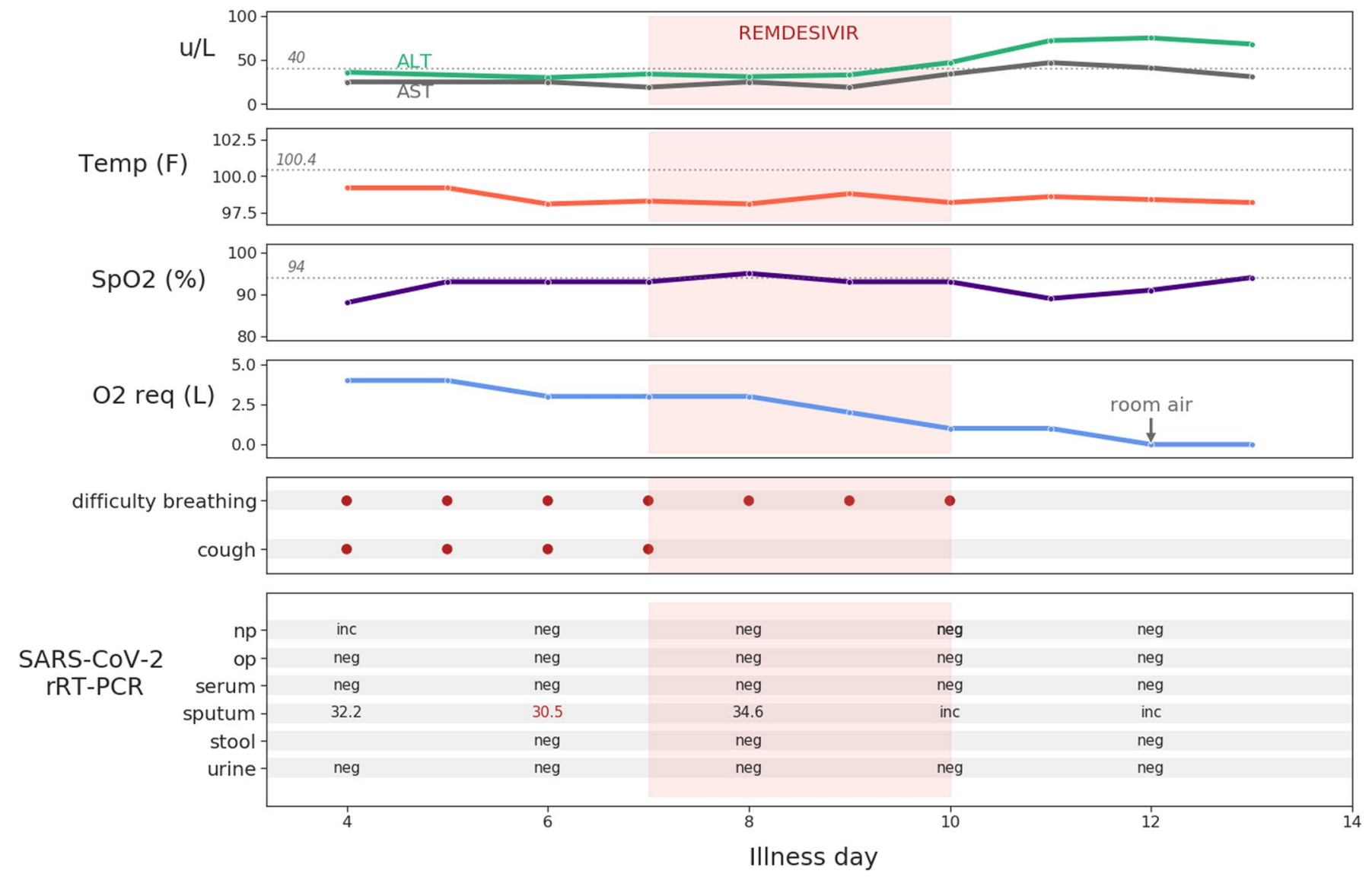

Extended Data Fig. 3 | Patient 8 laboratory and clinical values, symptoms, and SARS-CoV-2 rRT-PCR results during hospitalization. Aminotransferase levels, maximum daily body temperature, minimum daily oxygen saturation, maximum daily oxygen requirement, symptoms, and rRT-PCR results are shown for hospitalization. Pink shading indicates remdesivir administration. Cycle threshold (Ct) values are shown for positive rRT-PCR tests. Red text indicates decreasing $\mathrm{Ct}$ values. Patient has a chronic cough that had returned to baseline starting day 11. Abbreviations: AST, aspartate aminotransferase; ALT, alanine aminotransferase; SpO2, oxygen saturation; op, oropharyngeal; np, nasopharyngeal; neg, negative; inc, inconclusive. 


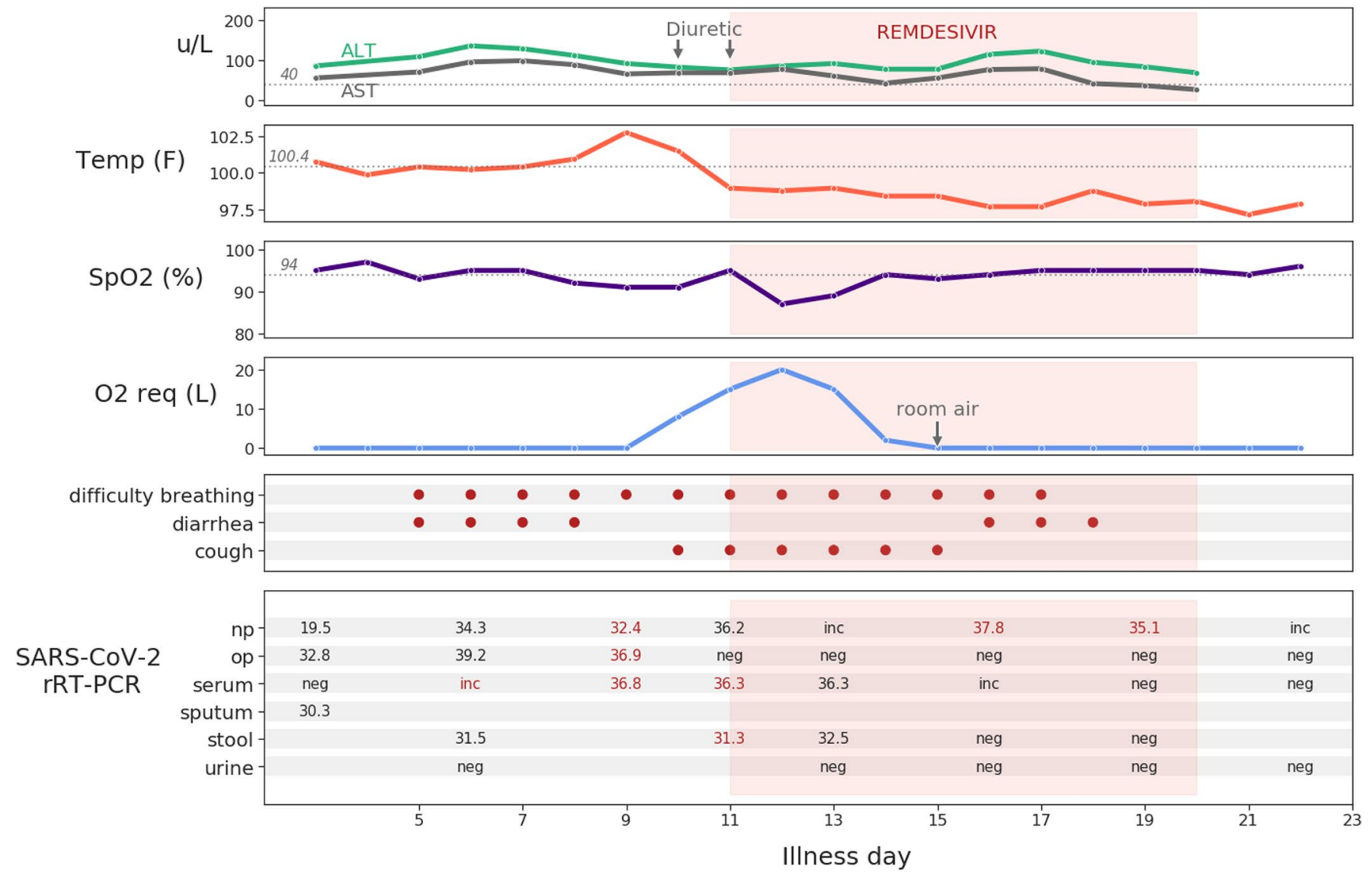

Extended Data Fig. 4 | Patient 9 laboratory and clinical values, symptoms, and SARS-CoV-2 rRT-PCR results during hospitalization. Aminotransferase levels, maximum daily body temperature, minimum daily oxygen saturation, maximum daily oxygen requirement, symptoms, and rRT-PCR results are shown for hospitalization. Pink shading indicates remdesivir administration. Cycle threshold (Ct) values are shown for positive rRT-PCR tests. Red text indicates decreasing Ct values. Abbreviations: AST, aspartate aminotransferase; ALT, alanine aminotransferase; SpO2, oxygen saturation; op, oropharyngeal; np, nasopharyngeal; neg, negative; inc, inconclusive. 


\title{
NATURE MEDICINE
}

$\mathrm{u} / \mathrm{L}$

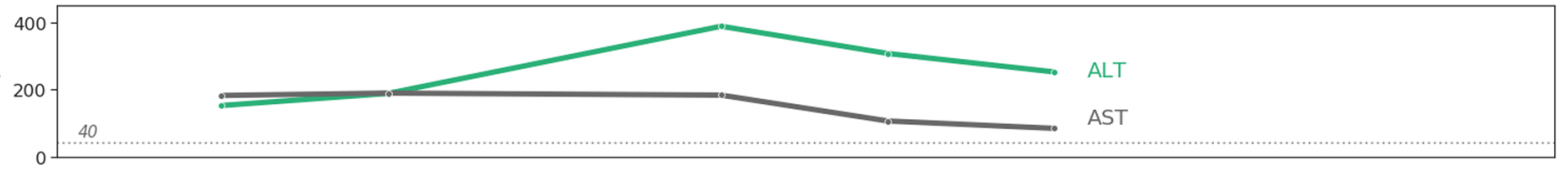

Temp (F)

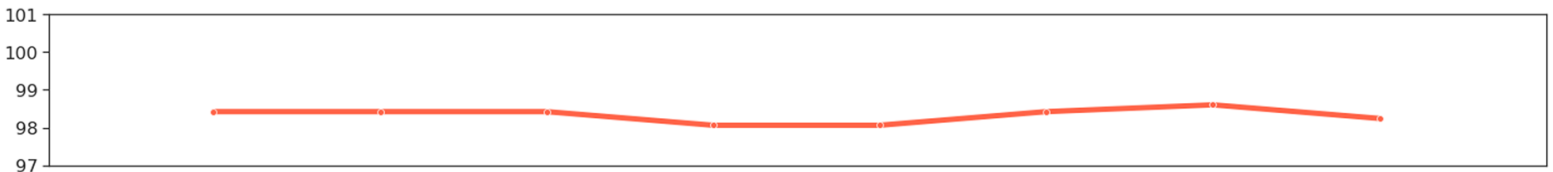

SpO2 (\%)
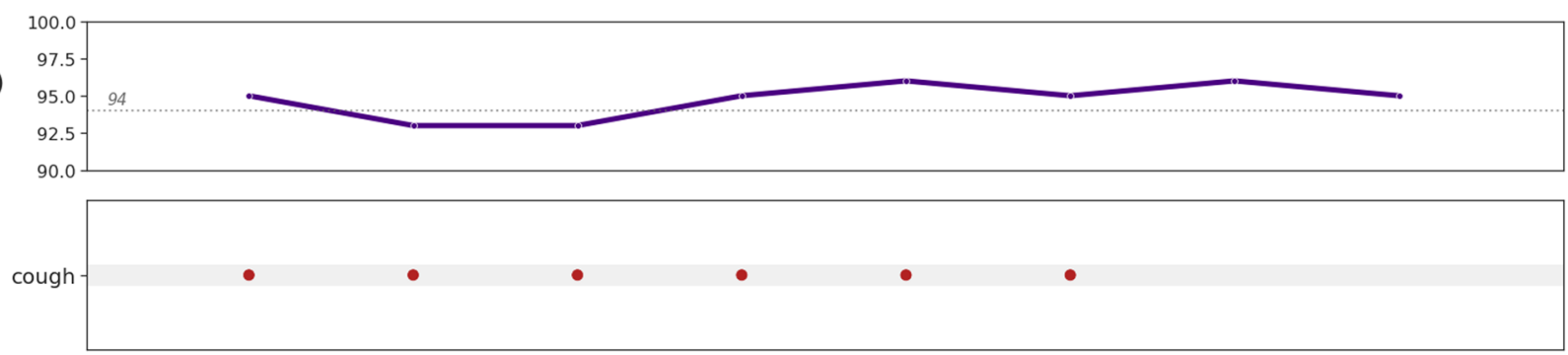

SARS-COV-2

rRT-PCR

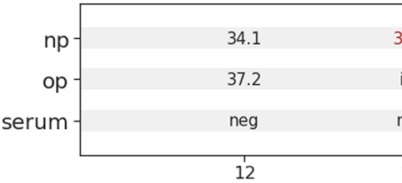

\begin{abstract}
33.8
inc

neg
\end{abstract}

35.7

37.3

13

14

15

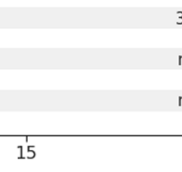

neg neg

neg neg

16

17

18

19

Illness day

Extended Data Fig. 5 | Patient 10 laboratory and clinical values, symptoms, and SARS-CoV-2 rRT-PCR results during hospitalization. Aminotransferase levels, maximum daily body temperature, minimum daily oxygen saturation, maximum daily oxygen requirement, symptoms, and rRT-PCR results are shown for hospitalization. Cycle threshold $(\mathrm{Ct})$ values are shown for positive rRT-PCR tests. Red text indicates decreasing $\mathrm{Ct}$ values. Abbreviations: AST, aspartate aminotransferase; ALT, alanine aminotransferase; SpO2, oxygen saturation; op, oropharyngeal; np, nasopharyngeal; neg, negative; inc, inconclusive. 


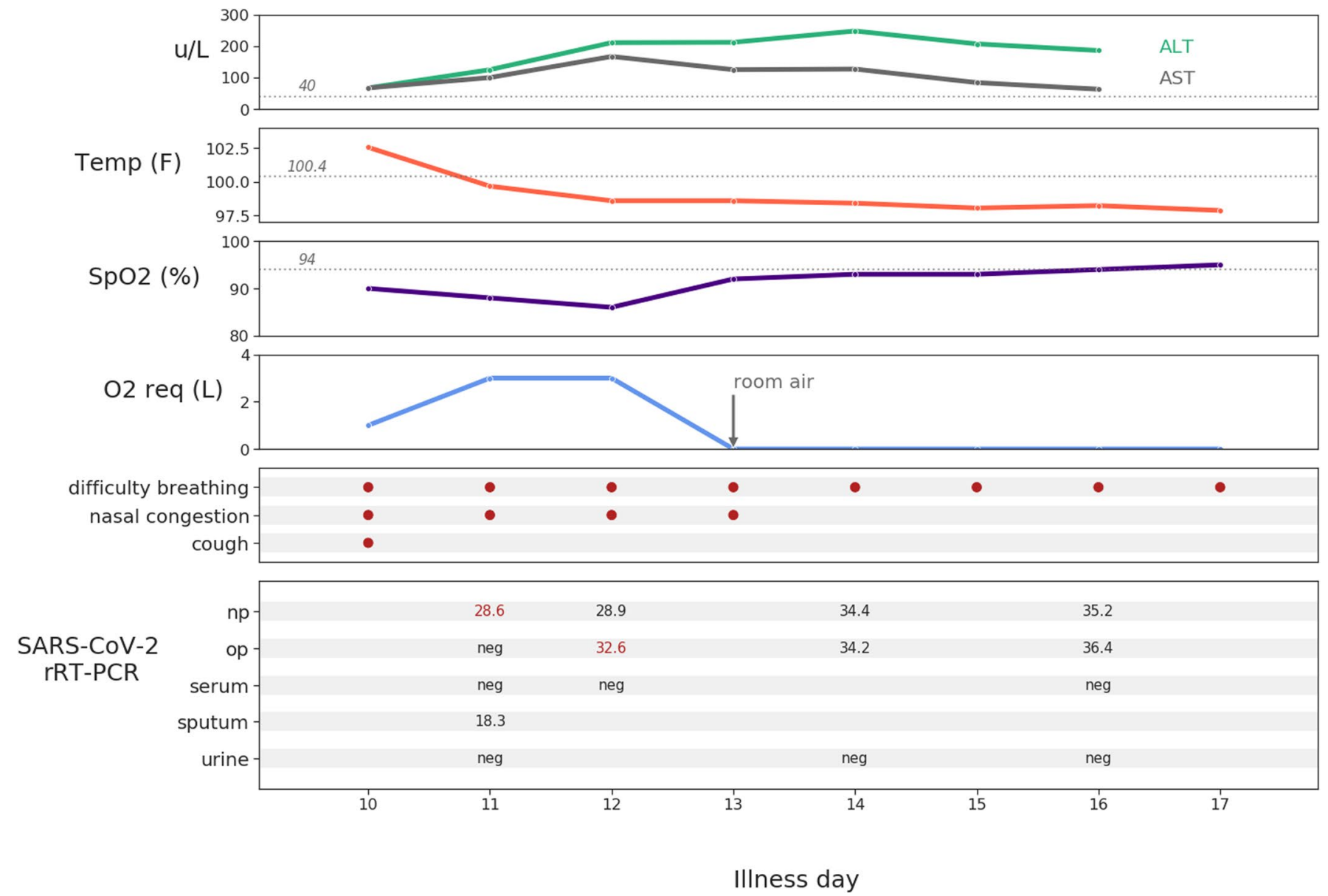

Extended Data Fig. 6 | Patient 11 laboratory and clinical values, symptoms, and SARS-CoV-2 rRT-PCR results during hospitalization. Aminotransferase levels, maximum daily body temperature, minimum daily oxygen saturation, maximum daily oxygen requirement, symptoms, and rRT-PCR results are shown for hospitalization. Cycle threshold (Ct) values are shown for positive rRT-PCR tests. Red text indicates decreasing $\mathrm{Ct}$ values. The patient had a positive nasopharyngeal swab on day 9 with Ct value of 35.1 (not shown). Abbreviations: AST, aspartate aminotransferase; ALT, alanine aminotransferase; SpO2, oxygen saturation; op, oropharyngeal; np, nasopharyngeal; neg, negative; inc, inconclusive. 


\section{NATURE MEDICINE}

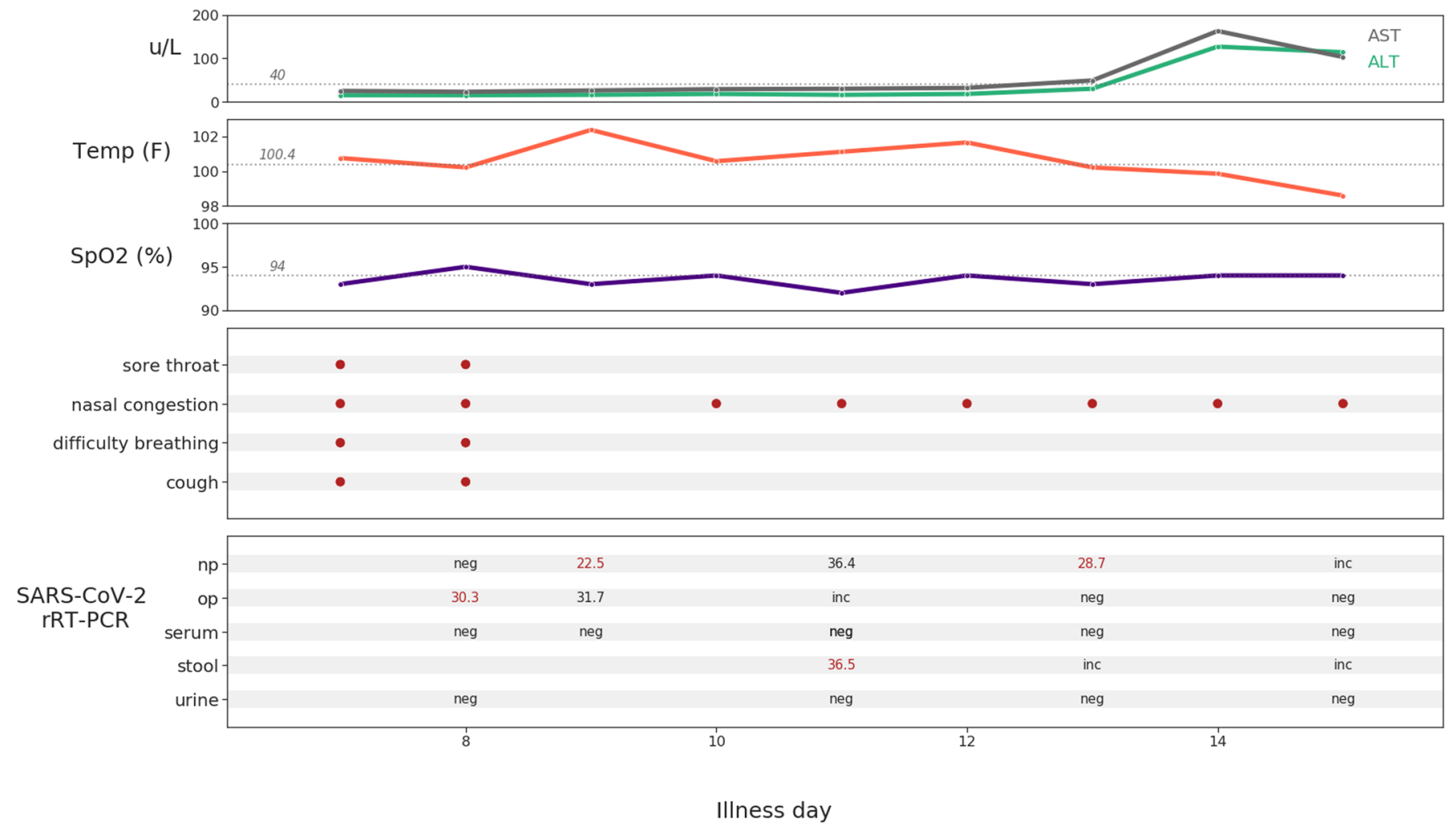

Extended Data Fig. 7 | Patient 12 laboratory and clinical values, symptoms, and SARS-CoV-2 rRT-PCR results during hospitalization. Aminotransferase levels, maximum daily body temperature, minimum daily oxygen saturation, maximum daily oxygen requirement, symptoms, and rRT-PCR results are shown for hospitalization. Cycle threshold $(\mathrm{Ct})$ values are shown for positive rRT-PCR tests. Red text indicates decreasing $\mathrm{Ct}$ values. Abbreviations: AST, aspartate aminotransferase; ALT, alanine aminotransferase; SpO2, oxygen saturation; op, oropharyngeal; np, nasopharyngeal; neg, negative; inc, inconclusive. 
SARS-CoV-2 average Ct values for patients with COVID-19 ( $n=12)$, by hosp. status and specimen type

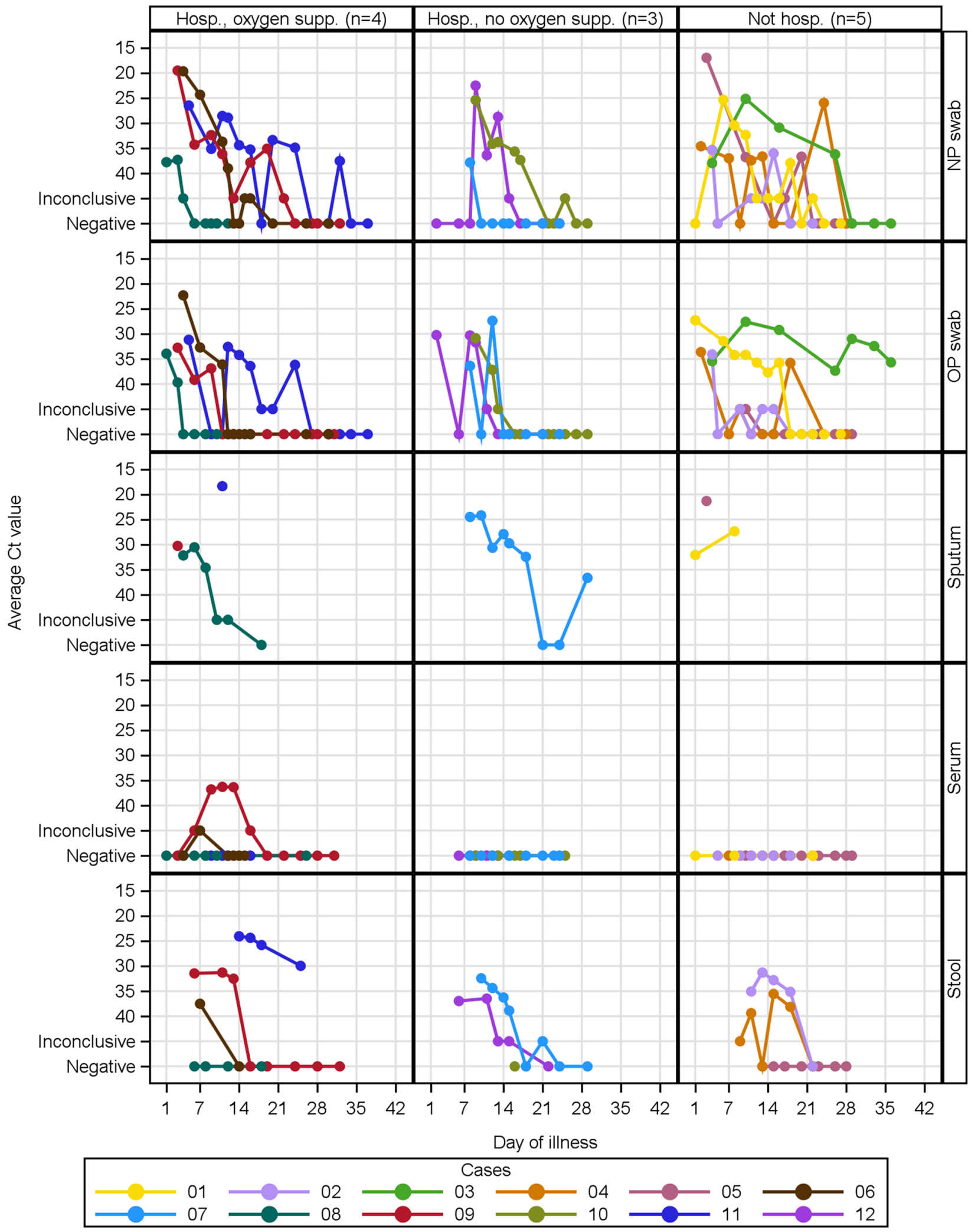

Extended Data Fig. 8 | See next page for caption. 
Extended Data Fig. 8 | SARS-CoV-2 average Ct values for patients with COVID-19, by hospitalization status and specimen type. For rRT-PCR-positive specimens, the average cycle threshold $(\mathrm{Ct})$ value was calculated from the $\mathrm{Ct}$ values of three targets on the gene encoding the $\mathrm{N}$ protein. 'Not detected' indicated rRT-PCR-negative specimens. Inconclusive specimens were depicted as such; average Ct values were not calculated for these specimens. Categories for illness severity included $\mathbf{a}$, not hospitalized, $\mathbf{b}$, hospitalized with no supplemental oxygen, and $\mathbf{c}$, hospitalized with supplemental oxygen. Specimen types depicted included nasopharyngeal (NP) swab, oropharyngeal (OP) swab, sputum, serum, and stool. Urine was not depicted because no specimens tested positive by rRT-PCR. Days are sequential from day of symptom onset (Day 1). 


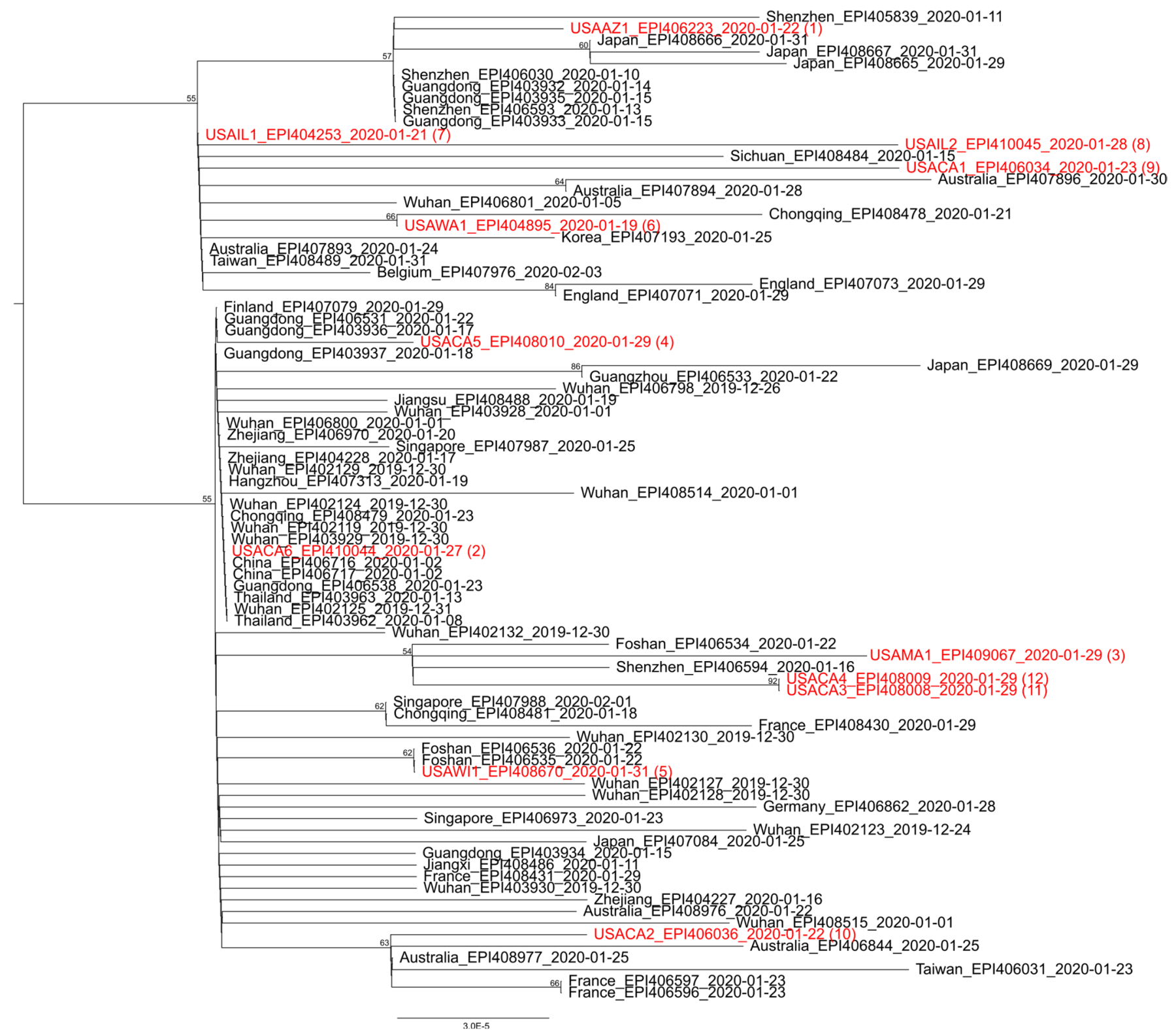

Extended Data Fig. 9 | Phylogenetic tree. A maximum-likelihood tree of SARS-CoV2 using selected full genome sequences from GISAID as of February 12 , 2020. Strains are denoted by country (city for China when available), GISAID accession ID, date of collection and Patient ID in parentheses. Tips in red denote sequences from US cases. Bootstrap values of statistical support (50\%) are shown as percentage equivalents. 


\section{natureresearch}

Corresponding author(s): Claire M. Midgley

Last updated by author(s): Apr 2, 2020

\section{Reporting Summary}

Nature Research wishes to improve the reproducibility of the work that we publish. This form provides structure for consistency and transparency in reporting. For further information on Nature Research policies, see Authors \& Referees and the Editorial Policy Checklist.

\section{Statistics}

For all statistical analyses, confirm that the following items are present in the figure legend, table legend, main text, or Methods section.

$\mathrm{n} / \mathrm{a}$ Confirmed

\ The exact sample size $(n)$ for each experimental group/condition, given as a discrete number and unit of measurement

$\triangle \square$ A statement on whether measurements were taken from distinct samples or whether the same sample was measured repeatedly

$\triangle \square$ The statistical test(s) used AND whether they are one- or two-sided

Xnly common tests should be described solely by name; describe more complex techniques in the Methods section.

$\bigotimes \square$ A description of all covariates tested

$\triangle \square$ A description of any assumptions or corrections, such as tests of normality and adjustment for multiple comparisons

$\varnothing$ A full description of the statistical parameters including central tendency (e.g. means) or other basic estimates (e.g. regression coefficient)

$\triangle \square$ AND variation (e.g. standard deviation) or associated estimates of uncertainty (e.g. confidence intervals)

$X$ For null hypothesis testing, the test statistic (e.g. $F, t, r$ ) with confidence intervals, effect sizes, degrees of freedom and $P$ value noted

$\triangle \square$ Give $P$ values as exact values whenever suitable.

Х $\square$ For Bayesian analysis, information on the choice of priors and Markov chain Monte Carlo settings

Х $\square$ For hierarchical and complex designs, identification of the appropriate level for tests and full reporting of outcomes

$\bigotimes \square$ Estimates of effect sizes (e.g. Cohen's $d$, Pearson's $r$ ), indicating how they were calculated

Our web collection on statistics for biologists contains articles on many of the points above.

\section{Software and code}

Policy information about availability of computer code

Data collection MinION data collected using MinKNOW 3.1.0.30. Raw data was basecalled with Guppy 3.2.8.

Reads were size-filtered using seqtk 1.3 and mapped to reference (Genbank MN908947.3) using minimap2 v2.17. Primer sequences were removed using BAMClipper 1.0.0. Variants were called with bcftools 1.9 and applied using vcf_mask_lowcoverage.pl (https:// github.com/CDCgov/SARS-CoV-2_Sequencing/tree/master/protocols/CDC-Comprehensive/scripts).

Data analysis

For phylogenetic analyses, sequencing data were analyzed with: Geneious Prime (BioMatters Inc., San Diego, CA) and Mega X (Kumar S, Strecher G, Li M, Knyaz C, and Tamura K (2018); MEGA X: Molecular Evolutionary Genetics Analysis across computing platforms, https:// doi.org/10.1093/molbev/msy096); and Fig Tree v1.4.3 (http://tree.bio.ed.ac.uk/software/figtree/, Andrew Rambaut). Other data were analyzed and visualized using Excel in Office 365, SAS 9.4, R 3.6.2, and Python 3.7.3

For manuscripts utilizing custom algorithms or software that are central to the research but not yet described in published literature, software must be made available to editors/reviewers. We strongly encourage code deposition in a community repository (e.g. GitHub). See the Nature Research guidelines for submitting code \& software for further information.

\section{Data}

Policy information about availability of data

All manuscripts must include a data availability statement. This statement should provide the following information, where applicable:

- Accession codes, unique identifiers, or web links for publicly available datasets

- A list of figures that have associated raw data

- A description of any restrictions on data availability

Virus sequences generated during the current study are available in the GENBANK repository (GenBank accession numbers: MN997409 (Pt 1), MT044258 (Pt 2), MT039888 (Pt 3), MT027064 (Pt 4), MT039887 (Pt 5), MT020880 (Pt 6), MN988713 (Pt 7), MT044257 (Pt 8), MN994467 (Pt 9), MN994468 (Pt 10$),$ MT027062 (Pt 11), MT027063 (Pt 12); GISAID numbers: 406223 (Pt 1), 410044 (Pt 2), 409067 (Pt 3), 408010 (Pt 4), 408670 (Pt 5), 407214 (Pt 6), 404253 (Pt 7), 410045 (Pt 8), 


\section{Field-specific reporting}

Please select the one below that is the best fit for your research. If you are not sure, read the appropriate sections before making your selection.

$\bigotimes$ Life sciences

Behavioural \& social sciences

Ecological, evolutionary \& environmental sciences

For a reference copy of the document with all sections, see nature.com/documents/nr-reporting-summary-flat.pdf

\section{Life sciences study design}

All studies must disclose on these points even when the disclosure is negative.

$\begin{array}{ll}\text { Sample size } & \begin{array}{l}\text { We have characterized the first } 12 \text { cases that were identified in the United States during a public health response; we included COVID-19 } \\ \text { patients confirmed by CDC during January 20-February 5, 2020; the size of this convenience sample was not predetermined, and we aimed to } \\ \text { describe the first cases as soon as possible. }\end{array}\end{array}$

Data exclusions No cases or data were excluded from this analysis.

Replication Data were verified by multiple authors, but this descriptive analysis on 12 cases was not replicated.

Randomization This is not relevant to our descriptive investigation performed during a public health response.

Blinding This was not relevant to our descriptive investigation performed during a public health response.

\section{Reporting for specific materials, systems and methods}

We require information from authors about some types of materials, experimental systems and methods used in many studies. Here, indicate whether each material, system or method listed is relevant to your study. If you are not sure if a list item applies to your research, read the appropriate section before selecting a response.

\begin{tabular}{l|l} 
Materials \& experimental sys \\
\hline$n / a$ & Involved in the study \\
\hline & $\square$ Antibodies \\
$\square$ & $\square$ Eukaryotic cell lines \\
$\square$ & $\square$ Palaeontology \\
$\square$ & $\square$ Animals and other organisms \\
$\searrow$ & $\square$ Clinical data
\end{tabular}

\begin{tabular}{l} 
Methods \\
\hline n/a Involved in the study \\
$X$ \\
$\square$ ChIP-seq \\
$\square$ Flow cytometry \\
$\square$ MRI-based neuroimaging
\end{tabular}

\section{Eukaryotic cell lines}

Policy information about cell lines

Cell line source(s)

Vero CCL-81 cells from ATCC

Authentication

ATCC uses morphology, karyotyping, and PCR based approaches to confirm the identity of human cell lines and to rule out both intra- and interspecies contamination. These include an assay to detect species specific variants of the cytochrome $C$ oxidase I gene (COI analysis) to rule out inter-species contamination and short tandem repeat (STR) profiling to distinguish between individual human cell lines and rule out intra-species contamination

Mycoplasma contamination

We test our cells for mycoplasma with Lookout PCR detection kit. Cell lines tested negative for mycoplasma contamination.

Commonly misidentified lines (See ICLAC register)

No commonly misidentified cell lines were used.

\section{Human research participants}

Policy information about studies involving human research participants

Population characteristics

Recruitment
Median age was 53 years (range: 21-68); eight patients were male.

Patients were identified as part of a public health response. The sample size was small, and results are not generalizable. 
